\title{
Tactile sensing in dexterous robot hands - review
}

\author{
Zhanat Kappassov ${ }^{\mathrm{a}, *}$, Juan-Antonio Corrales ${ }^{\mathrm{b}}$, Véronique Perdereau ${ }^{\mathrm{a}}$ \\ ${ }^{a}$ Institute of Intelligent Systems and Robotics, University of Pierre and Marie Curie, CC 173 - 4 Place Jussieu 75005, Paris, France \\ ${ }^{b}$ Institut Francais de Mecanique Avancee, Campus de Clermont-Ferrand les Cezeaux BP265 63175 AUBIERE Cedex, France
}

\begin{abstract}
Tactile sensing is an essential element of autonomous dexterous robot hand manipulation. It provides information about forces of interaction and surface properties at points of contact between the robot fingers and the objects. Recent advancements in robot tactile sensing led to development of many computational techniques that exploit this important sensory channel. This paper reviews current state-of-the-art of manipulation and grasping applications that involve artificial sense of touch and discusses pros and cons of each technique. The main issues of artificial tactile sensing are addressed. General requirements of a tactile sensor are briefly discussed and the main transduction technologies are analyzed. Twenty eight various tactile sensors, each integrated into a robot hand, are classified in accordance with their transduction types and applications. Previously issued reviews are focused on hardware part of tactile sensors, whereas we present an overview of algorithms and tactile feedback-based control systems that exploit signals from the sensors. The applications of these algorithms include grasp stability estimation, tactile object recognition, tactile servoing and force control. Drawing from advancements in tactile sensing technology and taking into consideration its drawbacks, this paper outlines possible new directions of research in dexterous manipulation.
\end{abstract}

Keywords: Tactile sensing, tactile sensors, robot hands, dexterous manipulation, tactile sensing application, review

\section{Introduction}

Autonomous dexterous manipulation, also known as in-hand object manipulation, is one of the much-desired key skills of industrial and social robots [1]. The development of autonomous dexterous robotic systems is a complex process of an interdisciplinary nature involving such diverse research fields as computer vision, force control, motion planning, grasping, sensor fusion, digital signal processing, human-robot interaction, learning and tactile sensing [2]. In this paper we address the issue of tactile sensing reviewing the current state-of-the-art tactile sensors and their applications in dexterous robot hands.

During the last decades, industrial robots have replaced humans in heavy, repetitive or/and unsafe manufacturing tasks [3]. The car, consumer electronics, and aerospace industries, to name only a few, have used pre-programmed robotic manipulators equipped with simple two-finger grippers in large scale production lines. Nevertheless, current manufacturing demands dictate a need for lower volume assembly of more customizable and variable products, requiring robots with higher adaptability, easy reconfigurability in software and hardware, more flexibility and more manipulation capabilities [4]. This need can be met by replacing grippers with multi-fingered dexterous robot hands

\footnotetext{
* Corresponding author

Email addresses: zhanat.kappassov@isir.upmc.fr (Zhanat Kappassov), juan.corrales@ifma.fr (Juan-Antonio Corrales), veronique.perdereau@upmc.fr (Véronique Perdereau)
}

that are able to grasp very different objects and even manipulate them with the use of fingers [1]. Dexterous robot hands are also essential in the new generation social and service robots which can replace humans in daily routines [5], and provide assistance to the elderly and the disabled. The incursion of robotics in domestic life presents new challenges to robotic design. Unlike industrial environments domestic spaces are typically unstructured which means that perception needs to be added to the robots' control strategies.

Among perception modalities, tactile sensing plays an important role in physical interactions, especially with human beings. Neuroscience has long demonstrated the importance of tactile feedback in human manipulation. Different studies have shown that people with anesthetized fingertips are unable to maintain a stable grasp [6], and children with deficient tactile sensing have difficulties in performing manipulation tasks [7]. Tactile sensors provide robots with information about physical contact, whereby autonomous robot hands can operate in unstructured environments and manipulate unknown objects [8]. At the same time, the availability of sensory information to the robot ensures its safe operation in direct human-robot interaction applications.

In traditional industrial approaches control of robot end-effectors is achieved by embedding prior knowledge about articulated object and environment into the control algorithm. Robot hands are thus able to manipulate only known objects and work in a structured environment, 
which means they are less adaptive to unexpected events. To overcome these limitations, an approach based on active exploration, which relies on data from tactile sensors, can be implemented to let robot hands explore objects and run control actions when unexpected events occur. Only a few approaches use tactile feedback inside autonomous control schemes [8].

Artificial tactile sensors in robotic applications are represented by pressure profile sensing arrays, force-torque sensors, and dynamic tactile sensors [9]. Information acquired from artificial sensing systems can be used for finding contact locations, reconstructing and recognizing object shape, and measuring contact forces and temperature.

Even though tactile sensory information is an essential element in the process of manipulation, technology and research in artificial tactile sensing is not developed as well as other perception modalities [10]. Promising new technological advances in tactile sensors based on microelectromechanical systems [11] and organic transistors [12], have not been applied yet to robotic devices.

Currently research is focused on developing new tactile skins, covering robot hands with tactile sensors and investigating new algorithms and approaches for using tactile information in autonomous manipulation. New techniques that use tactile sensing information include object recognition and exploration, grasp stability estimation, force control, tactile servoing and slip detection.

This paper presents a thorough review of the most recent advances in robotic tactile sensing. Previous review articles have mostly concentrated in tactile hardware dealing with tactile sensing technologies for robot hands [13], for minimal invasive surgery [14], for biomedical applications [15], slip detection in hand prostheses [16], robotic tactile skins [17] and large area tactile skins [18]. This paper will review the techniques for handling tactile data in robotic manipulation applications covering approaches and applications of tactile sensors in the control of multifingered robotic hands. The paper is organized as follows: Tactile sensing technologies are given in Section 2. Integration of the sensors with robot hands and tactile data acquisition are reviewed in Section 3. This is followed by a survey of computational techniques that use tactile information to control the robot hands. These techniques include grasp stability estimation 4.1, object recognition 4.2, force control 4.4 and tactile servoing 4.3. A summary of the conclusions appear in Section 5.

\section{Tactile Sensing Technologies}

Information about interaction properties can be acquired from proprioceptive (intrinsic) sensors, such as joint angle sensors with actuator torque sensors, and cutaneous (extrinsic) tactile sensors [17]. Even though, intrinsic sensors can give approximate information about interaction force as shown elsewhere [2], extrinsic tactile sensors give much more precise and multi-modal information about interaction properties [19]. Thus, tactile sensors can be de- fined as a tool that can evaluate a given property of an object through physical contact between the hand and the object [20]. When a tactile sensor is represented by an array, each sensing element of the sensor is referred differently in robotics literature, e.g. sensing cell, taxel or tactel.

Tactile sensors meet the following task-related requirements of in-hand manipulation [10]:

1) Response. In collision avoidance [21] and human-robot interaction tasks, tactile sensors must provide information about the presence of contact and measure the strength of contact force, respectively.

2) Exploration. During exploration, tactile sensors should provide information about: surface properties from measurements of a texture, hardness, and temperature [22]; structural properties from shape [23]; and functional properties from detection of contacts and vibrations [24].

3) Manipulation. In autonomous manipulation tasks, tactile data is used as a control parameter in: slip detection; estimation of grasp stability[25]; contact point estimation, surface normal and curvature measurement [26]; tangential and normal forces measurements for achieving stable grasps [27]; and contact force measurements for fingertip force control [28].

Depending on the task, the sensor has different design specifications, which were first determined by Harmon [29]. The basic design criteria for tactile sensors have been previously reported in [17] for humanoid robots, in [15] for biomedical engineering, in [16] for prosthetic hands, and in [18] for manufacturing and large tactile system implementation. In autonomous manipulation applications, tactile sensors meet requirements for object characterization and identification (e.g. they estimate the compliance, thermal and textural properties) and for manipulation (e.g. they control the force applied to the object) [19].

The most important design criteria for tactile sensors with application in manipulation tasks are summarized in Table 1 and discussed in following:

1) Requirements on spatial resolution of a tactile sensing array depends on both the size of the objects to be recognized and the location of the sensor on a robot hand. A rather high spatial resolution is desirable in inhand object manipulation [30] or tactile servoing [31] tasks, whereas in the cases when high sensitivity or high frequency response are desirable, e.g. reactive force control [32], the spatial resolution is limited by for the following reasons. A higher spatial resolution unavoidably leads to a longer acquisition time [33], a larger number of wire connections and a stronger sensitivity to external electromagnetic noises. The first two consequences are straightforward, high resolution requires a large number of sensing cells, which in turn causes longer processing time. These sensing cells also require more wire connections. The highest limit of sensitivity is given by the minimum detectable variation of the measured signal. As sensing cells become smaller the sensitivity to external electro-magnetic noises and crosstalk increases. Thus, the sensitivity degrades be- 
cause the level of noise can become comparable with the signal. By considering these pros and cons, the requirements on spatial resolution can vary for different parts of a robot hand. It was previously investigated that the resolution on the fingertips should be as high as $1 \mathrm{~mm}$ since the fingertips are mostly involved in fine manipulation [29]. In the current state of the art, fingertip tactile sensors integrated with robot hands have a spatial resolution of around $5 \mathrm{~mm}$ [34], [35]. On less sensitive parts of a robot hand like the palm, the spatial resolution decreases up to $5 \mathrm{~mm}$ as stated in [17]. Requirements for spatial resolution can be omitted when only slippage is of importance, e.g. automatic grasping using vibrations to achieve stable grasp [32] and slip-detection with center-of-pressure tactile sensor [36].

2) Sensitivity in the tactile sensors is given by the smallest detectable variation in pressure/force. A small detectable variation means a high sensitivity. High sensitivity is very important in manipulation tasks with fragile and deformable objects as in [37] or [38]. However, the range from the minimum to maximum detectable pressure/force, i.e. dynamic range, shrinks with the increase of the sensitivity of a tactile sensor, which is caused by the technology used in the structure of the current sensors. An area of sensing cells the sensor also causes contradiction between sensitivity and spatial resolution as was discussed above.

Dahiya et al. [17] impose following requirements. The sensitivity on the fingertips should be not less than $1 \mathrm{mN}$, while a dynamic range of $1000: 1$ is desirable.

3) Requirements for frequency response highly depend on the application. In general, tactile sensors can be dynamic or static [9]. If the hand is required to detect vibrations during slippage, the frequency response should be as high as the vibration frequencies occurring during a slippage [16], [32], [38], [39]. In human hands, the detectable vibration frequencies vary from $5 \mathrm{~Hz}$ to $50 \mathrm{~Hz}$ and from $40 \mathrm{~Hz}$ to $400 \mathrm{~Hz}$ for different afferents [6]. Thus the frequency response of a dynamic tactile sensor should be at least $400 \mathrm{~Hz}$, i.e. the sampling rate must be at least $800 H z$ according to Nyquist-Shannon sampling theorem. When only spatial resolution is of importance (e.g. tactile object recognition [40]), then the frequency response is not restricted by the response time. On the contrary, when measurements of vibrations are used to prevent a slippage [41], to detect a contact of a grasped object with an environment [38] or to recognize a texture of a surface [22], then the response time of a sensor becomes crucial. The frequency response (bandwidth) is limited by the softness (elasticity) of a tactile sensor. The use of soft materials, that are used to increase surface friction, causes phase delay in propagation of the waves of the mechanical vibrations that occur at the point of contact.

4) Hysteresis and memory effect ideally should be as low as possible. Tactile sensing arrays incorporating flexible foam in their structure it unavoidably leads to an elastic behavior of the sensors. Once the sensor is pressed and released, the flexible foam first compresses and then regains its form but not immediately (hysteresis effect) and sometimes not to the previous shape (memory effect). Moreover, the sensor could be covered by a soft material, e.g. silicon rubber as in [42]. The advantage of using flexible materials is the increase of a contact friction. However, sensitivity and frequency response of a sensor may degrade with the increase of flexibility. Though, reading devices can have high sampling rate, a sensor may have significant hysteresis, which reduces dynamic response [9]. The memory effect could be avoided by use of a thinner foam, which in turn decreases the dynamic range, since the maximum charge (in capacitive sensors) that can be stored is proportional to the thickness of the foam. This maximum charge represents the largest detectable force.

5) Wiring of tactile sensors should not affect the workspace of robot hands [18]. Integration of a high number of tactile sensors in the robot hand is challenging due to wiring constraints. As an example, in [43] a multimodal tactile sensor is installed as a complete fingertip with bulky backside instead of distal and middle phalanges. Shielding and smart wiring should guarantee minimum sensitivity to noise and minimum tactile cross-talk. Use of serial communication protocol decreases the number of connection wires as in iCub skin [44], but it increases the sampling rate.

6) A sensor itself should be flexible so it can be attached to any type of robot hand [44], unless the sensor is designed as a complete part of a robot hand, as for example the 3D-shaped tactile sensing fingertip in [34].

7) Surface properties of tactile sensors, such as mechanical compliance and surface friction coefficient should fit to various manipulation tasks. Elastic material with given friction coefficient and compliance can cover tactile sensors. If the contact sensing surface has very low friction, then the hand must apply high normal forces to keep the object stable, which can lead to breaking the object [34]. However, the low friction of the sensor surface is needed in tactile exploration procedures [31].

8) A robust sensor design should guarantee that the sensor can withstand highly repetitive usage without its performance being affected. The sensor should endure normal as well as lateral forces.

\subsection{Tactile sensor types}

Change of capacitance, resistance, optical distribution, electrical charge can be used in the sensing systems [45], [46].In the robotics literature, these different ways to construct the sensing systems are referred as transduction of contact information [10]. And the types of tactile sensors vary depending on the transduction.

In the following we describe the basic types of tactile sensors and their transduction methods. The advantages and disadvantages of each sensor type are given in Table 2 . 
Table 1: Design criteria: pros and cons.

\begin{tabular}{|c|c|c|c|}
\hline Criteria & pros & cons & Application \\
\hline $\begin{array}{l}\text { High spatial } \\
\text { resolution }\end{array}$ & $\begin{array}{l}\text { A smaller objects can be recognized } \\
\text { and features with a higher precision } \\
\text { can be extracted. }\end{array}$ & $\begin{array}{l}\text { A smaller sensitivity and a longer } \\
\text { processing time. }\end{array}$ & $\begin{array}{l}\text { Contact pattern } \\
\text { recognition, fine } \\
\text { manipulation. }\end{array}$ \\
\hline $\begin{array}{l}\text { High } \\
\text { sensitivity }\end{array}$ & $\begin{array}{l}\text { Detection of a rather small change } \\
\text { of a contact force. }\end{array}$ & $\begin{array}{c}\text { Dynamic range of the sensor } \\
\text { shrinks, spatial resolutions } \\
\text { decreases. }\end{array}$ & $\begin{array}{l}\text { Light touch } \\
\text { detection and } \\
\text { fragile object } \\
\text { manipulation. }\end{array}$ \\
\hline $\begin{array}{l}\text { High } \\
\text { frequency } \\
\text { response }\end{array}$ & $\begin{array}{c}\text { A rather fast response to the } \\
\text { changes in the level of the contact } \\
\text { force }\end{array}$ & $\begin{array}{c}\text { Spatial resolution and dynamic } \\
\text { range decrease }\end{array}$ & $\begin{array}{l}\text { Detection of a slip } \\
\text { and texture } \\
\text { recognition. }\end{array}$ \\
\hline $\begin{array}{l}\text { Low } \\
\text { hysteresis }\end{array}$ & High frequency response & $\begin{array}{l}\text { Degrease of the sensor's surface } \\
\text { friction and dynamic range. }\end{array}$ & $\begin{array}{l}\text { Detection of a slip } \\
\text { and texture } \\
\text { recognition. }\end{array}$ \\
\hline $\begin{array}{l}\text { Low number } \\
\text { of wire } \\
\text { connections }\end{array}$ & $\begin{array}{l}\text { The workspace of robot hands does } \\
\text { not change. }\end{array}$ & $\begin{array}{l}\text { Decrease of the frequency response } \\
\text { (in case of using serial data } \\
\text { communication). }\end{array}$ & $\begin{array}{l}\text { Dexterous } \\
\text { manipulation }\end{array}$ \\
\hline $\begin{array}{l}\text { High surface } \\
\text { friction }\end{array}$ & $\begin{array}{l}\text { Insuring stable grasp without } \\
\text { applying high forces. }\end{array}$ & $\begin{array}{l}\text { Imped tactile exploration } \\
\text { procedure. Reduces the frequency } \\
\text { response of the sensor (in case of } \\
\text { using soft paddings). }\end{array}$ & Grasping \\
\hline
\end{tabular}

\subsubsection{Piezoresistive sensors}

The piezoresistive effect is a physical process during which electrical resistance changes when the material is mechanically deformed (Figure 1(a)) [45]. Materials possessing this effect are called piezoresistors [51].

There are several technologies for artificial tactile sensing based on piezoresistive materials: Force Sensing Resistors (FSR), pressure-sensitive conductive rubber, piezoresistive foam, and piezoresistive fabric.The simplest way to incorporate tactile sensing via discrete components is by using FSRs [9] and they are widely used in positioning devices such as joysticks [52]. Piezoresistive rubber is a composite material made by mixing non-conductive elastomer with homogeneously distributed electrically conductive carbon particles [53], [47]. Figure 1(b) shows the structure of conductive rubber at nano-scale level [48]. Sensors based on conductive rubber with multilayer structures as in [49], [39], [35], [54] (Figure 1(c), 1(d)) may suffer from delamination of top layers. This can be avoided by using a single layer of the conductive rubber with a stitched array of wires in orthogonal orientations as in [55]. Another method of designing tactile sensing arrays using the conductive rubbers incorporates a non-flexible pattern of the electrodes on one layer and piezoresistive rubber on a second layer (Figure 1(e) [34]. Some of the sensors and components are commercially available from Interlink [56] and the Tekscan [57] (FSRs), Weiss Robotics [58] (rigid tactile sensors based on carbon enriched silicone rubber), Inaraba [59] (pressure conductive rubber), Eeonyx [60] (piezoresistive fabric), ATi industrial automa- tion [61] (Force/Torque sensors).

It is worth mentioning that currently developed tactile sensors based on pressure sensitive rubber and organic transistors, such as the ones used in the bionic skin [62], are exceptionally thin and highly flexible. Conductive rubbers used in piezoresistive sensors have a nonlinear forceresistance characteristic (please refer to sensor calibration plot in [31]). As a consequence of using elastic materials, the sensors have severe hysteresis. The sensitivity in the piezoresistive sensors may decrease due to wearing and tearing off, since the resistance of the conductive rubber does not depend on deformation only but also on thickness. Moreover, materials used in the piezoresistive sensors could change their properties due to variation of the temperature and moistness [45].

Piezoresistors also suffer from lower repeatability:after multiple deformations, an elastic material may never regain its initial form. Some of the piezoresistive sensing arrays are also fragile to shear forces, e.g. Weiss tactile sensors [58]. In spite of these drawbacks, a number of robot hands incorporate piezoresistive tactile sensing arrays, since the sensors are relatively simple to manufacture, can be flexible and many commercial solutions exist. Compared to capacitive sensors that will be discussed in the next section, the piezoresistive sensors are more robust (not completely) to electro-magnetic noises.

\subsubsection{Capacitive sensors}

Capacitive sensors consist of two conductive plates (Figure 2(a)) separated by a compressible dielectric material 


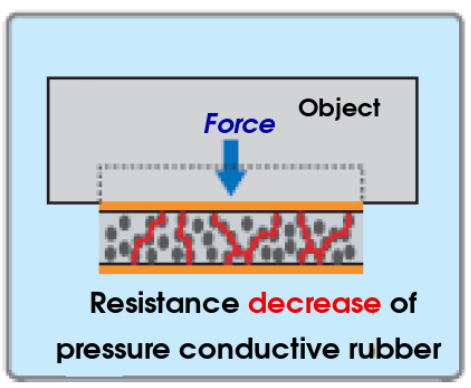

(a)

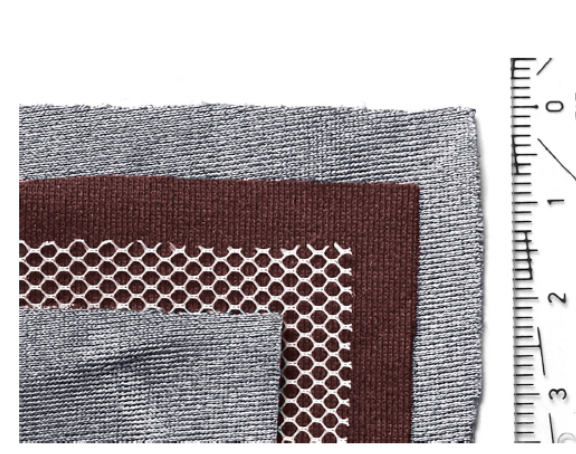

(d)

(d)

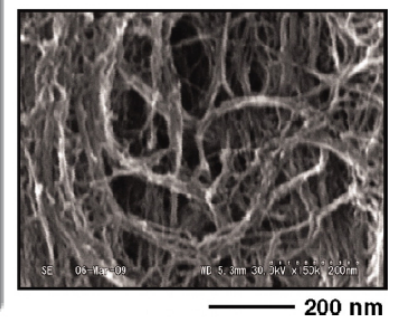

(b)

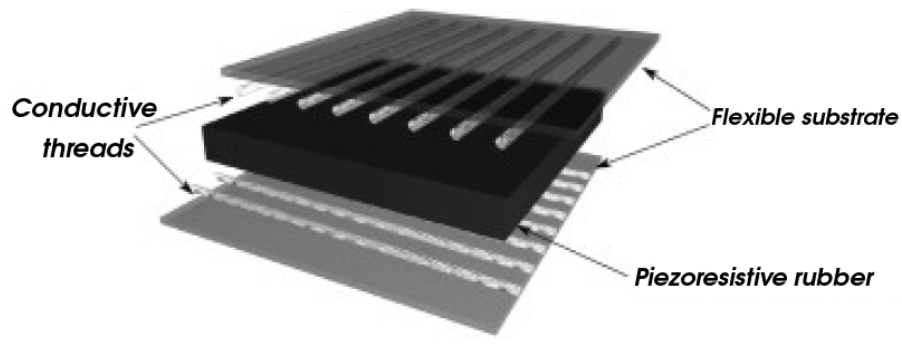

(c)
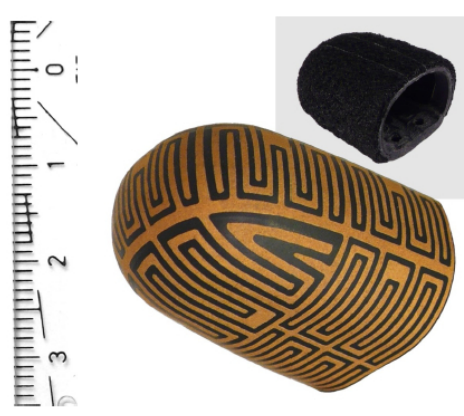

(e)

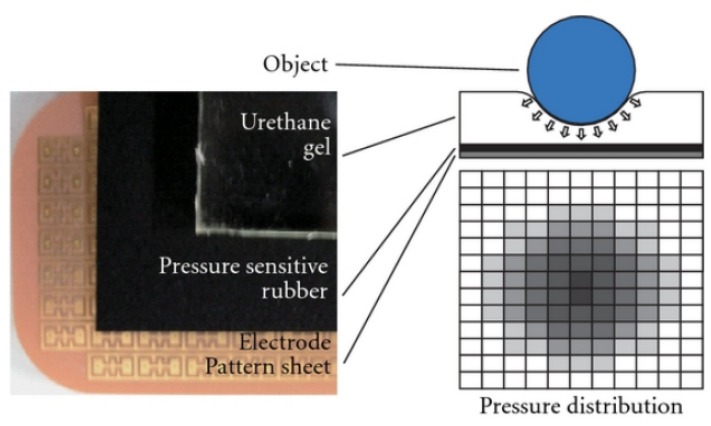

(f)

Figure 1: Piezoresistive Tactile Sensor Arrays: (a) illustration of resistance changes in conductive rubber [47],(b) nano-scale image of conductive rubber [48], (c) structure of piezoresistive tactile array [49], (d) piezoresistive fabric tactile sensor [50], (e) schematic of electrode layer of the 3D-shaped tactile sensor [34], (f) tactile image of a piezo-resistive pressure sensor array [35].

(Figure 2(b)). When the gap between plates changes under the applied forces, the capacitance is also changed. Besides normal forces, the shear forces can be calculated by the sensor with the use of embedded multiple capacitors [63]. Pressure sensing arrays can be constructed by overlapping row and column electrodes isolated from each other by elastic dielectric [33]. Sensitivity to small forces can be achieved by using more compressible elastic materials or thin sensors. As a flexible foam between two plates gets thinner than a smaller charge in the sensor could be measured that is in turn means a higher sensitivity.

Capacitive technology is very popular among the sensing transducers and it has been widely used in robotic applications [17]: for example, in tactile the skin (Figure 2(c)) for the iCub humanoid robot [44], in the PR2 robot grippers [38], with the multifingered "Allegro" robot hand [64], and with the Robotiq robot gripper [65].

There are commercial capacitive pressure sensing arrays such as "DigiTacts" from Pressure Profile Systems (PPS) [66] and capacitance-to-digital-converter (CDC) chips such as "AD7147" from Analog Devices [67].

The major disadvantages of capacitive sensors are susceptibility to electro-magnetic noise, sensitivity to temperature, non-linear response (please refer to the plot with response of excited taxel in [68]), and hysteresis. Their advantages include a higher frequency response relatively to piezoresistive sensors. Since capacitive technologies are

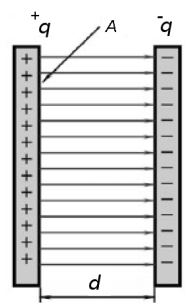

(a)

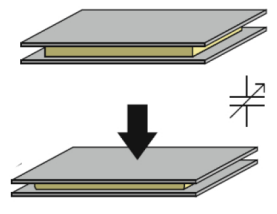

(b)

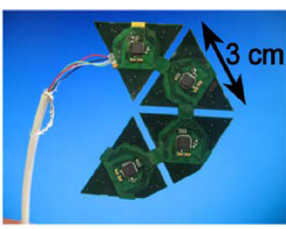

(c)
Figure 2: Capacitive Tactile Sensing Technology: (a) capacitance of a parallel plate capacitor depends on distance between plates $d$ and area of the plates $A$ ( $q$ is the stored charge) [45]; (b) two conductive plates are separated by an elastic dielectric - as force is applied, the distance between the plates reduces, changing the capacitance [9]; (c) mesh of triangle shape capacitive sensors for the palm of the $\mathrm{iCub}$ humanoid robot [44].

used in every day life applications, as for example touch screens, this type of tactile sensing have been well investigated and used in robotics and especially in robot hands.

\subsubsection{Piezoelectric sensors}

The piezoelectric effect (Figure 3(a)) is described as electrical charge generation in the crystalline material due to deformation caused by applied force/pressure [45]. The piezoelectric effect is produced in quartz crystals, as well as in human-made ceramics and polymers, such as polyvinyli- 


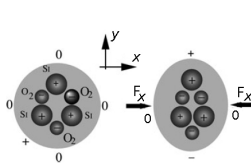

(a)

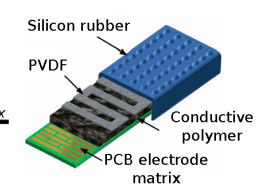

(b)

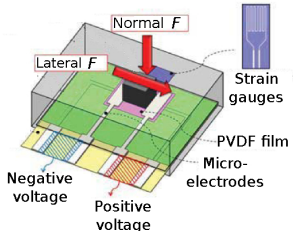

(c)
Figure 3: Piezoelectric Tactile Sensing: (a) the piezoelectric effect an applied force causes rearrangement of positive $S i$ and negative $\mathrm{O}_{2}$ particles leading to an increase of potential [45]; (b) a tactile sensing array based on the piezoelectric effect with electrodes on the bottom layer, piezolectric material in the middle and rubber on the top [24], (c) schematic model of a piezoelectric sensing tactel [71].

dene fluoride (PVDF) [69]. A piezoelectric tactile sensor can be created with the PVDF film strips embedded into a rubber material. Piezoelectric materials, being restricted for dynamic measurements and used in ultrasonic-based sensors, are suitable for dynamic tactile sensing [17], [33]. Among other piezoelectric materials, PVDF polymer has features such as flexibility and chemical stability, which makes it preferable for use in touch sensors. Seminara et al. [69] conducted research on PVDF electro-mechanical design of tactile sensors with frequency range of $1 \mathrm{~Hz}$ to $1 \mathrm{kHz}$. Goger et al. [24] developed a combined dynamic/static tactile sensor (Figure 3(b)) based on PVDF polymer and piezoresistive foam from Weiss Robotics for a fluidic robot hand [70]. Chuang et al. [71] developed a flexible tactile sensor based on piezoelectric film with structural electrodes for grasping an object of unknown weight (Figure 3(c)).

Piezoelectric materials have high bandwidth up to $7 \mathrm{kHz}$ as reported in [24]. These materials have faster dynamic response than capacitive sensors. Their disadvantages include fragility of electrical junctions, temperature sensitivity [52] and they are suitable for dynamic measurements only.

\subsubsection{Quantum Tunnel Effect Sensors}

Quantum Tunnel Composite (QTC) sensors can change their properties from insulators to conductors under compression [17]. QTC sensors are more technologically advanced compared to piezoresistive and capacitive sensors. The metal particles in QTC get so close to each other that quantum tunneling (of electrons) takes place between the particles. Using QTC material, Zhang et al. 2013 [72] (Figure 4) developed a flexible tactile sensor for an anthropomorphic artificial hand with capability of measuring shear and normal forces. The sensor has sensitivities of $0.45 \mathrm{mV} / \mathrm{mN}$ in $\mathrm{x}$ - and y-directions and of $0.16 \mathrm{mV} / \mathrm{mN}$ in z-directions, and dynamic ranges up to $8 N$ in $\mathrm{z}$ - and $\mathrm{y}$-directions and $20 \mathrm{~N}$ in $\mathrm{x}$-direction. QTC-based tactile sensors [73] were integrated with previous versions of the Shadow robot hand [74] and used in the tactile glove for the Robonaut hand [75]. The sensors have linear response (please refer to sensor outputs w.r.t normal force in [72])

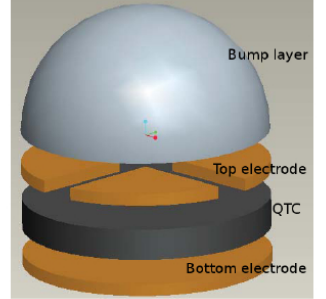

(a)

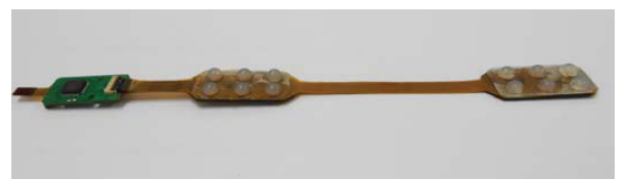

(b)

Figure 4: Quantum Effect Tactile Sensing: (a) structure of a tactel of the QTC based tactile sensing array with capability of measuring shear and normal forces [72]; (b) the flexible tactile sensing array for a finger of an anthropomorphic robot hand with the tactels that can measure shear forces [72].

and a dynamic range starting from 0 to $22 N$ which outperform the piezoresistive sensor with a maximum force of $5 \mathrm{~N}$ [31] in terms of the dynamic range. These sensors suffer from wear and tear of and, therefore, their sensitivity decreases as in the case of the piezoresistive sensors. To the best of our knowledge, for the tactile sensing materials within this category, there are no commercial products that are designed for use with robot hands.

\subsubsection{Optical sensors}

Optical sensing is based on optical reflection between mediums with different refractive indices. Conventional optical tactile sensors consist of an array of infrared lightemitting diodes (LEDs) and photo detectors (Figure 5(a)). The intensity of the light is proportional to the magnitude of the pressure [45]. Optical sensors can also be made sensitive to shear forces, e.g. Yussof et al. [37] developed an optical three-axis tactile sensor for the fingertips of a two-fingered hand (Figure 5(b)). The sensor consists of 41 sensing elements made from silicon rubber, a light source, an optical fiber-scope, and a current charged coupled device (CCD) camera. With the optical tactile sensor, the hand is capable of manipulating a light paper box (Figure 5(c)). Kampmann et al. [76] embedded fiber optic sensors to a multi-modal tactile measuring system of a three-fingered robot gripper (Figure $7(\mathrm{~d})$ ). Xie et al. developed a flat $3 \times 3$ optical tactile sensor array (Figure $5(\mathrm{~d}))$ with elements of the sensor that are magnetic resonance compatible for use in Magnetic Resonance Imaging [77]. Johnson et al. [78] proposed a novel "GelSight" tactile sensor to capture surface textures using an elastomer coated with a reflective membrane and a camera with resolution of up to 2 microns. (Figure 5(e)). A fingertip with a "GelSight" (Figure 5(f)) tactile sensor can measure the surface roughness and texture, the pressure distribution, 


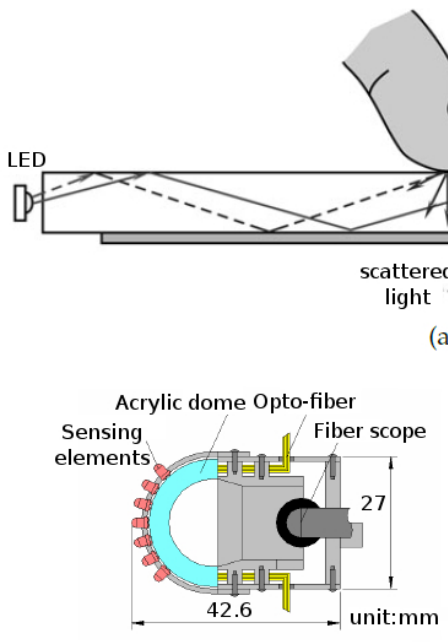

(b)

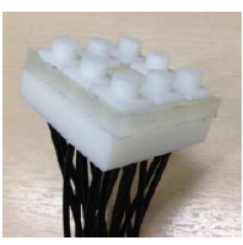

(d)

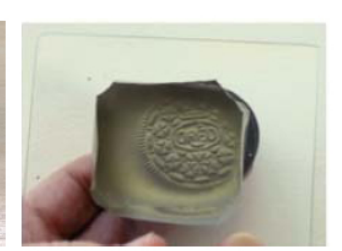

(e)

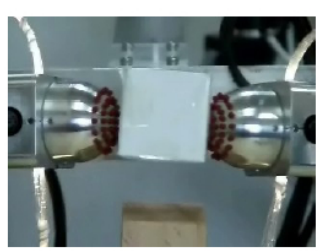

(c)

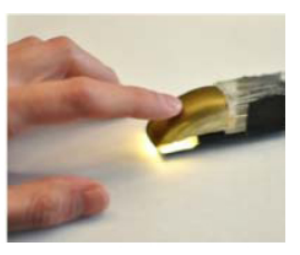

(f)
Figure 5: Optical Tactile Sensors: (a) an optical tactile transducer based on the principle of frustrated total internal reflection [45], (b) a structure of optical three-axis tactile sensor: a displacement of a sensing element fixed on flexible finger surface causes changes in light propagation in opto-fibers [37], (c) fingers with the sensitive optical sensors manipulating a light paper box [37], (d) photo of an optical 3 x 3 tactile array with magnetic field compatibility [77], (e) "GelSight" optical sensor consisting of a piece of clear elastomer coated with a reflective membrane senses the shape of the cookie surface [79], (f) finger configurations of the "GelSight" sensor [79].

and even a slip [79]. Another example of an optical tactile sensor with transparent elastomer material is presented in [80], where an LED and a photo-diode distant from each other are placed against a reflecting (contact) planar surface. When surface deforms it causes changes in reflected beams. Similar concept is used in the OptoForce sensors [81]. These sensors are based on the use of infrared light to detect deformation of the contact surface, which in turn transforms to force. The forces in three dimensions are estimated from measurements of four photo-diodes that surround one infrared source. The reflecting surface has a semi-spherical shape.

Sensors within this category have good spatial resolution, sensitivity, high repeatability and immunity from electro-magnetic interference [15]. The disadvantages of these tactile sensors are their relatively big size, highpower consumption and high computational costs [10].

\subsubsection{Sensors based on barometric measurements}

Tactile sensors within this group use pressure transducers that have been long used for measuring the pres-

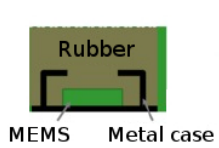

(a)

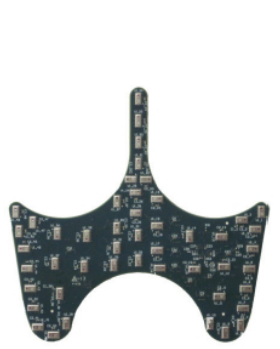

(c)

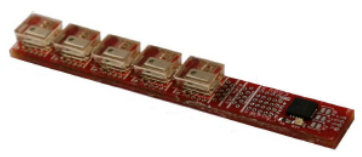

(b)

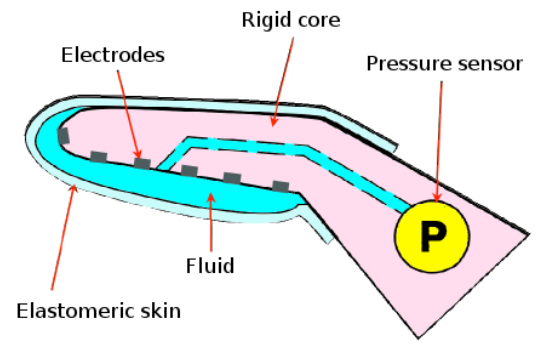

(d)

Figure 6: Sensors based on barometric measurements: (a) the structure of a tactile sensing cell with a barometer and silicon rubber (b), the TakkStrip tactile array of these cells [87], (c) custom shaped array of the pressure sensing barometers of the iHY hand [42], (d) micro-vibration sensing system based on a fluid pressure sensor of the BioTac tactile sensor [83].

sure in liquids and air [45]. Use of liquid inside a tactile sensor allows getting high frequency response and deformability of the sensor at the same time. A liquid is used as propagation media for vibrations, which are represented by changes in pressure value. This approach takes advantage of the conventional pressure sensors, as for example the digital barometer [82]. Wettels et al. [19] introduced the sensing system that incorporates electroconductive fluid to produce both constant and dynamic signals (Figure 6(d)). Micro-vibrations, caused by either motion over textured surface or slippage at any contact point, propagate as sound waves through the liquid media to a pressure transducer [83]. The bandwidth of the sensor is $1 \mathrm{kHz}$, which makes the sensing system well suitable for slip detection applications. The sensor is embedded in the multi-modal biomimetic $囚$ BioTac fingertip sensor from SynTouch LLC [84].

In [85], no liquid is used as a propagation media, a barometer is instead molded within a silicon rubber in each tactel. The rubber acts as a membrane (Figure 6(a)) . Once the rubber is deformed due to the contact with an environment, it causes changes in the pressure values of the barometer. Using the same digital barometer, Odhner et al. [42] developed a tactile sensor array (Figure 6(c)) with a spatial resolution of around $(3-5 \mathrm{~mm})$, sensitivity $1 m N$, and dynamic range up to $4.9 N$ for a three-fingered robot hand [42].

Sensors involving liquid and barometers have high frequency response [86]. Sensors with silicon rubber and barometer are low-cost, but has low frequency response [85] as a result of the elasticity of the silicon rubber. Hence, the use of a liquid as a propagation media is more suitable when frequency response is of importance. 


\subsubsection{Multi-modal Tactile Sensors}

To match the human hand's different types of tactile sensing modalities (thermal, fast adapting and slow adapting afferents) [6] as close as possible, a robot hand should be equipped with multi-modal tactile sensors. Current multi-modal tactile sensing systems incorporate static pressure distribution arrays, dynamic tactile sensors, thermal sensors, and proximity sensors. The BioTac fingershaped sensor array (Figure 7a,b) provides information about the contact forces, microvibrations, and temperature produced during contact with external objects [19]. Some tactile sensors have the ability to sense dynamic and static contact forces since they have been constructed using a combination of piezoresitive and piezoelectric materials. Examples of such material include piezoresistive rubber with PVDF (Figure 3(b)) [24] that is integrated with an anthropomorphic fluidic hand [70] and pressure variable resistor ink with PVDF that is integrated with a four fingered robot hand [88]. Another hybrid sensing system with similar combination of dynamic and static transducers combines carbon micro-coil touch sensor and a force tactile sensor [89]. Hasegawa et al. integrated proximity and pressure sensors on the fingertip (Figure $7(\mathrm{c})$ ) to enhance autonomous grasping [90]. Optical sensors also found their application in the multi-modal approach. A three-fingered robot gripper described in [76] incorporates optical sensors and combines measurements of absolute forces by strain gauge sensors, dynamic forces by piezoelectric sensors, and force distribution by fiber optic sensors, as shown in Figure $7(\mathrm{~d})$. Unlike the above multimodal sensors, in which locations of sensing units are known, a sensing system of a robot fingertip proposed by Hosoda et al. [91] has random distribution of the sensing units. Similar to [24], the sensing system consists of piezoresistive and piezoelectric sensors to measure static forces and vibrations. The piezoelectric sensors are placed at a skin layer and inside the fingertip thus giving possibility to measure internal vibrations. The only drawback of the multimodal tactile sensors is their size.

\subsubsection{Structure-borne sound tactile sensors}

Vibrations and waves in solid structures are summarized by the term "Structure-borne sound" [92]. In manipulation tasks, structure born sounds occur at the initial contact of a manipulated object with the environment or during the slippage. Accelerometers and microphones can be used as detecting devices. In pick-and-place manipulation tasks, these structure born sounds can serve as indicators to trigger the placement of the object by the manipulator. Romano et al. [38] use a high sensitive 3-axis accelerometer in the base of PR2 robot gripper in order to detect the contact of the object with the table and to release the object. Earlier, Kyberd et al. [32] integrated a microphone with an anthropomorphic prosthetic hand for automated grasping.

Sensors within this group have wide bandwidth, but are suitable for dynamic measurements only. However, in

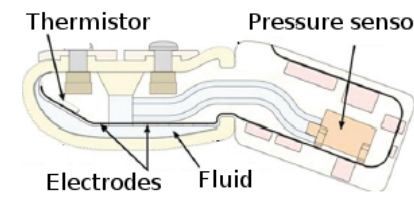

(a)

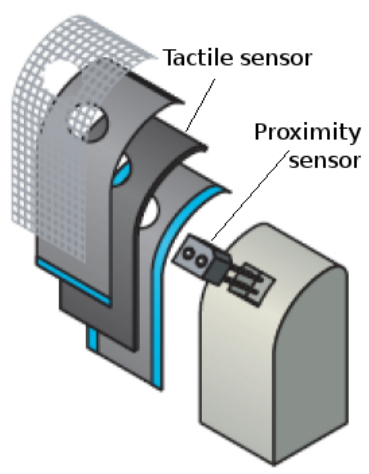

(c)

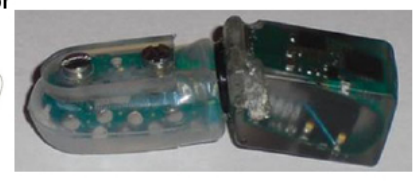

(b)

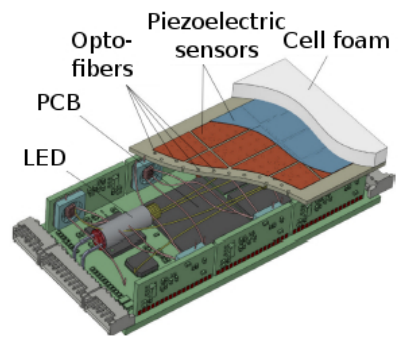

(d)
Figure 7: Multimodal Tactile Sensors: (a) schematic of the biomimetic BioTac tactile sensor with 19 electrodes, fluid pressure sensor and thermometer [84], (b) photo of the multimodal BioTac tactile sensor, (c) combined tactile-proximity sensor that can measure both the distance to an object and the contact pressure [90], (d) drawing of a multi-modal tactile sensing module consisting of optical and piezoresistive sensors [76].

a close proximity of an object, it is possible to estimate the distance to the object by comparing a level of an environmental acoustic noise and a level of noise within the sensor as has been shown by Jiang et al. [93]. The presented concept of the sensor is based on Seashell Effect - increase of a level of noise in cavities due to resonance of sound waves and intrinsic resonance frequency of a cave. The sensor incorporates a cavity and a microphone located inside the cavity. The cavity has its own resonance frequency that depends on both the structure of the cavity and the distance from an object to be grasped.

Data stream coming from tactile sensors has different physical meanings for different transduction technologies. In general it can be dynamic or static according to the time response and may represent an array of data, vector or scalar value. Hence, data acquisition from different sensors has its own approaches as discussed in the following section.

\subsection{Tactile data types and acquisition}

Force torque sensors installed on the fingertips of a robot hand provide with force and torque values in each direction in $\mathbb{R}^{3}$. A contact point location can be estimated from these forces and torques as long as the shape of the fingertip is known [26]. The measured forces and torques can then be used for force control (Section 4.4) and in haptic object recognition (Section 4.2). 
Table 2: Tactile sensing types: advantages and disadvantages of major sensor types. Abbreviations for the names: PRes. - piezoresistive sensors, Cap. - capacitive, PEl. - piezoelectric sensor, Opt. - optical sensors, BarS. - sensors based on barometric measurements, MultiM. - multimodal sensors, SoundS. - structure borne sound sensors.

\begin{tabular}{|c|c|c|}
\hline Type & Advantages & Disadvantages \\
\hline PRes. & $\begin{array}{l}\text { Many commercial } \\
\text { solutions exist, } \\
\text { simpler for } \\
\text { manufacturing, } \\
\text { can be flexible. }\end{array}$ & $\begin{array}{c}\text { Non-linear response, } \\
\text { temperature and } \\
\text { moistness } \\
\text { dependence, fatigue, } \\
\text { permanent } \\
\text { deformation, } \\
\text { hysteresis }\end{array}$ \\
\hline Cap. & $\begin{array}{c}\text { A number of } \\
\text { commercial } \\
\text { solutions, can be } \\
\text { flexible, may have } \\
\text { higher bandwidth } \\
\text { than PRes. } \\
\end{array}$ & $\begin{array}{c}\text { Susceptibility to } \\
\text { electro-magnetic } \\
\text { noise, sensitivity to } \\
\text { temperature, } \\
\text { non-linear response, } \\
\text { hysteresis. } \\
\end{array}$ \\
\hline PEl. & $\begin{array}{l}\text { Very high } \\
\text { bandwidth }\end{array}$ & $\begin{array}{c}\text { Temperature } \\
\text { dependence, dynamic } \\
\text { sensing only }\end{array}$ \\
\hline QTC & $\begin{array}{l}\text { Linear response, } \\
\text { higher dynamic } \\
\text { range (w.r.t Cap. } \\
\text { and PRes) }\end{array}$ & $\begin{array}{l}\text { More complex for } \\
\text { manufacturing (w.r.t } \\
\text { in Cap. and PRes) }\end{array}$ \\
\hline Opt. & $\begin{array}{l}\text { High spatial reso- } \\
\text { lution, high } \\
\text { sensitivity, } \\
\text { repeatability, } \\
\text { immunity to EM } \\
\text { noise }\end{array}$ & $\begin{array}{l}\text { Bulky, high-power } \\
\text { consumption, high } \\
\text { computational costs }\end{array}$ \\
\hline $\begin{array}{l}\text { BarS. } \\
\text { (fluid) }\end{array}$ & $\begin{array}{l}\text { High bandwidth, } \\
\text { high sensitivity, } \\
\text { temperature and } \\
\text { moistness } \\
\text { independence }\end{array}$ & $\begin{array}{c}\text { Low spatial } \\
\text { resolution }\end{array}$ \\
\hline SoundS. & High bandwidth & Dynamic sensing only \\
\hline
\end{tabular}

Tactile sensors with fast response (such as accelerometers, microphones, piezoelectric and capacitive technology based sensors, and barometers with fluid media) provide information about vibrations at the contact point (see Figure $8(\mathrm{~b}))$. Information about vibrations can be further used for slip detection and haptic object exploration (Sections 4.2.2 and 4.1.2). The dynamic response of the tactile sensing arrays is limited by the sampling rate of reading devices. In [39], the sampling rate of data acquisition board is $10 \mathrm{kHz}$, while in [9] the signal bandwidth is limited by the sampling rate $(300 \mathrm{~Hz})$ of a commercial capacitance-to-digital-converter [67]. Figures 9(a) and (b) show schematic diagrams of reading devices for dynamic capacitive and piezolectric PVDF sensors.

Information from pressure sensing tactile arrays can be

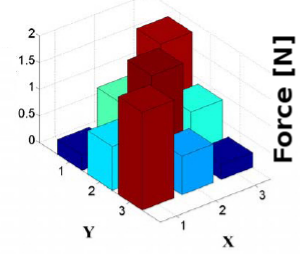

(a)

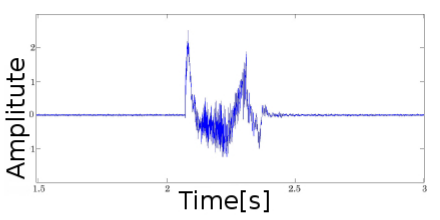

(b)

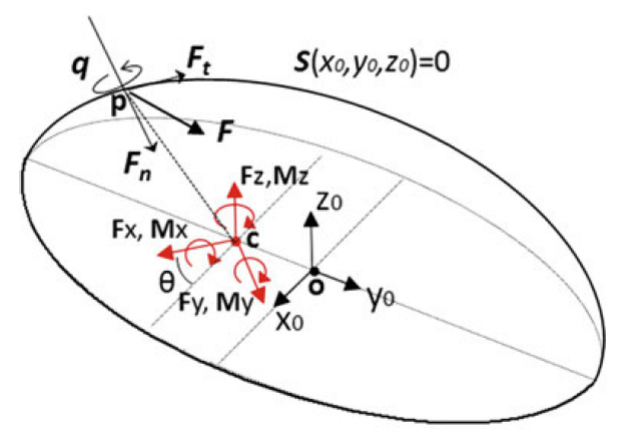

Ellipsoidal Fingertip

(c)

Figure 8: Tactile sensing signal types: (a) a two-dimensional pressure distribution of a tactile sensing array, where the sensing tactels are located on $x y$ plane and force/pressure is measured along $z$-axis [77]; (b) dynamic tactile signal from a single tactel or from an ensemble of tactles, which can be acquired during a slippage [24]; (c) 6 DoF force/torque sensor measurements in the ellipsoid-shape fingertip [26] including normal forces in each direction $F_{i}$ of the Cartesian space, torques $M_{i}$, contact point $P$, forces and torques at contact point $F$ and $q$.

treated as a gray-scale image in computer vision [40] (see figure $8(\mathrm{a})$ ). Although some tactile arrays may have tactels (Figure 4(a)) that can measure pressure in the threedimensional space as in [72], the value of each tactel in most of the current tactile sensors is proportional to applied normal pressure only. Tactile sensor arrays provide information about contact shape and pressure distributions [41].

In capacitive and piezoresistive sensors, data from each tactel can be acquired either directly, which means that a high amount of wires is required, or by using a multiplexing circuit (Figure 10(c)), which decreases twofold the number of wire connection.

Piezoresistive tactile arrays consist of a common electrode, of sensing electrodes that are arranged as a matrix, and of conductive rubber in between. Pressing on the sensor's surface provides an image of the applied pressure profile [53]. Figure 1(f) illustrates the image of the sensing array which is produced when a spherical object is pressing the tactile surface. Tactile images can be used for contact pattern recognition [94], grasp stability estimation [95], object classification [49], and tactile servoing [31].

For tactile sensing arrays, data acquisition involves the usage of analog to digital converters (Figure $9(\mathrm{~d})$ ) as well 


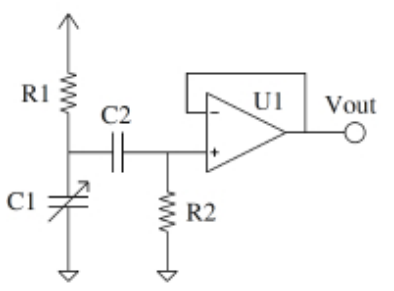

(a)

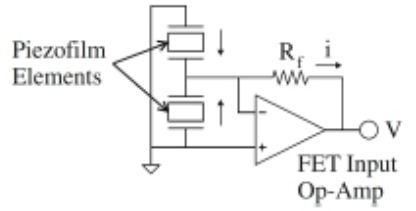

(b)

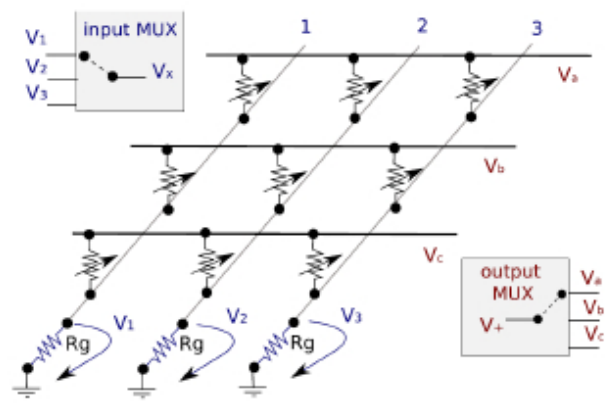

(c)

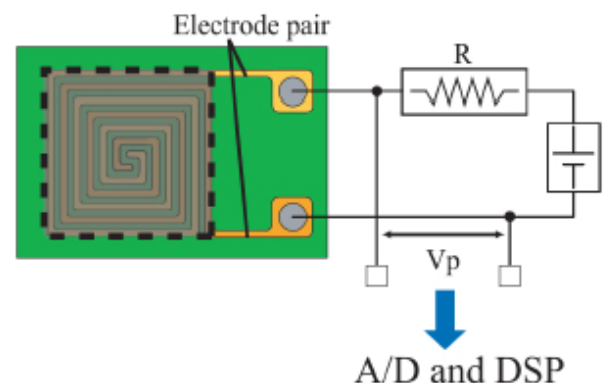

(d)

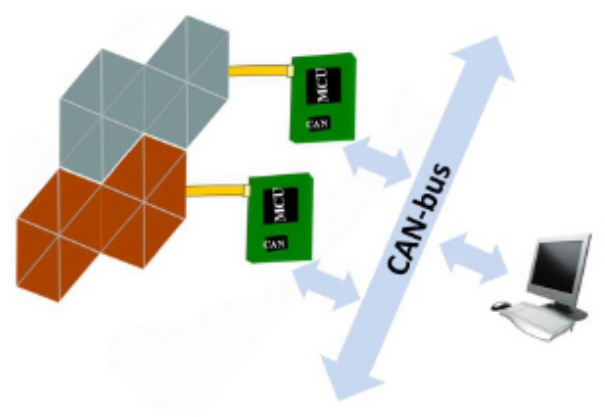

(e)

Figure 9: Tactile sensing reading circuits: (a) the condenser microphone circuit for capacitive sensors [9]; (b) a circuit for utilizing piezoelectric PVDF film as a stress rate sensor [9]; (c) signal conditioning and voltage multiplexing for a $3 \times 3$ tactile sensing array [49]; (d) the voltage-divider circuit for a pressure conductive rubber [39]; (e) network structure of the iCub tactile sensing skin using CAN-bus for connecting tactile sensing patches, 12 tactels in each patch, with a main processing unit [44]. as of microprocessing units for polling each tactel [18]. The capacitance of capacitive tactile sensors can be measured by commercial CDCs chips. The CDC chips can include $I^{2} C$ serial interface. Digital barometers such as the absolute digital pressure sensor "MPL115A" [96] that is used in the iHY robot hand [42] has also $I^{2} C$ serial interface. Communication with processing units can be realized via different transmission protocols (e.g. controller area network (CAN), and universal serial bus (USB), RS232). In iCub skin [44], local measurements are sent by an on-board processing unit over a CAN bus (Figure 9(e)). Multimodal tactile sensing data in the BioTac sensor [19] is acquired by the PIC microprocessor and sent to the host processing unit over serial peripheral interface (SPI). In order to minimize memory use of micro-processing units, data coming from sensors can be preprocessed by signal conditioning circuits, which can be implemented as system on chip (SOC) or system in package (SIP) [18].

In some specific applications, for example in fast reaction to slip [24], signals from tactile sensors can be analyzed and processed within a controller without sending information to the host computer. In most of the applications, middleware and high-level software installed onto the main processing unit is used to compute acquired data and control the system. For these purposes versatile open source and commercial robot control platforms are available: in [30], robot operating system (ROS) [97] is used to control Shadow robot hand [98], robot platform (YARP) [99] is used to control iCub humanoid robot [100]; the controlling operating system dSPACE from dSPACE Co. is used in [39] to control high speed-robot hand [101] in realtime and $\mathrm{C}++$ libraries of open Robot control software (OROCOS) [102]. Among open source robot control platforms, ROS is the most widely used and supports both simulation (Gazebo simulator) and control of the Shadow hand, the Barret hand and many other manipulators and robots.

\section{State of the art tactile sensor integration with robot hands}

In this section we review the existing robot hands equipped with tactile sensors and discuss several issues related to the integration process.

\subsection{Issues related to the shape of the attachment surface}

Mounting tactile sensors on the palm, a jaw grippers or on fingers with flat surfaces is relatively straightforward, one of the simplest ways involving using a double side tape. Figure 10(a) shows an experimental setup containing the Shadow Hand and the Tekscan tactile sensing system (Model 4256E), which was used for contact shape recognition [94]. In another manipulating setup, off-the-shelf capacitive arrays have been installed on the fingertips of the four-fingered "Allegro" robot hand (Figure 10(b)). Figure 10(c) illustrates the Robotiq adaptive 

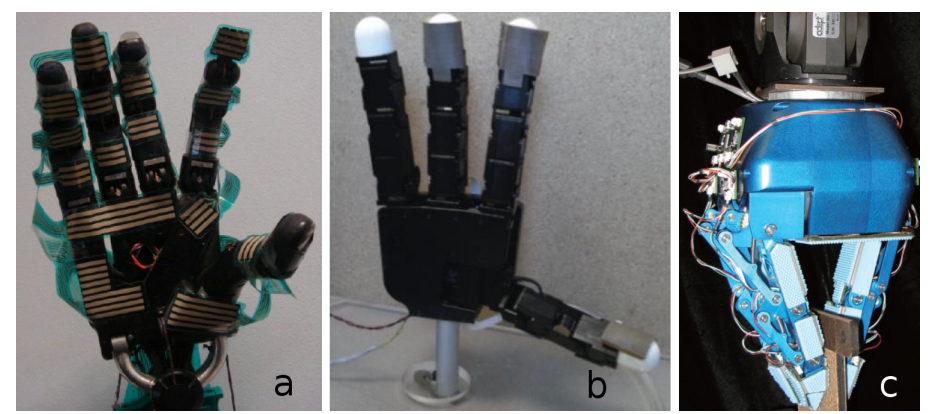

Figure 10: Simple integration of tactile sensing arrays: (a) the Tekscan tactile sensing system consisting of 349 taxels with the Shadow robot hand [94], (b) the Allegro robot hand with PPS RoboTouch capacitive arrays [64], (c) the Robotiq adaptive gripper with sensor suite installed on the contact surface [65]

gripper covered by capacitive pressure sensing arrays used for the recognition of the type of the slip [65]. Attaching tactile sensors on fingers and fingertips is a complex process as curved surfaces with small radius of curvature have to be taken into account. Tactile sensors should be either: a) flexible and appropriately shaped to envelop a given surface, as in iCub tactile fingertip sensors (Figure 11(a, b) [103]; b) rigid and shaped as an attachment part, e.g. [34] or [104] where a 3D-shaped tactile sensing array and an ellipsoid F/T sensor (Figure 11(c) and (e) ) replace the fingertips of the Shadow robot hand [98]. In another version of the Shadow robot Hand with the integrated BioTac multimodal tactile sensor, each finger loses one DoF (Figure $11(\mathrm{~d}))$, - the sensor is as big as the two last links, distal and middle phalanges of the human index finger.

The shape of the links of the fingers in robot hands is different from the shapes of human finger phalanxes. The proximal and middle links of fingers in artificial robot hands have a smaller contact surface than those of humans, a fact that significantly decreases the sensing area and causes difficulties with attachment. Figure 12 shows the difference between sensing areas on the middle and proximal links of a human finger and a robot finger. Current artificial tactile sensors are not as flexible as human skin and cannot cover the empty space between the links for closing the finger of robot hands.

\subsection{Wiring issues}

A key issue in tactile sensing array integration is the amount of wires required to read and transmit the data from the sensing arrays. Any increase in the number of tactels in tactile sensing array causes an increase either in the number of wires or/and on the time needed for data acquisition from sensors. A serial data communication can be used to reduce the number of connections. For example, in the iCub skin, communication was implemented through I2C serial bus, where only four wires were connected to the PCB of the sensing array [44]. However, serial access of data is slower than parallel access. In $\mathrm{iCub}$,

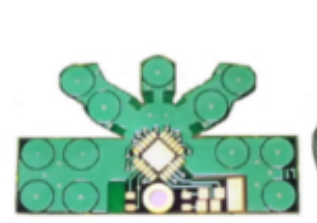

(a)

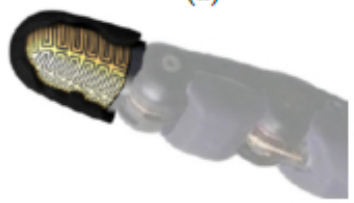

(c)

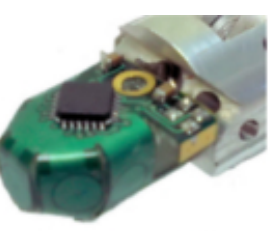

(b)

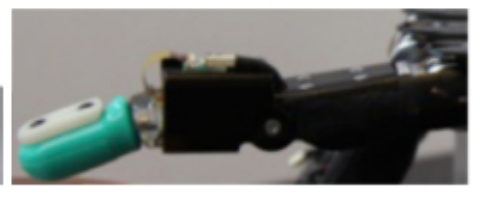

(d)

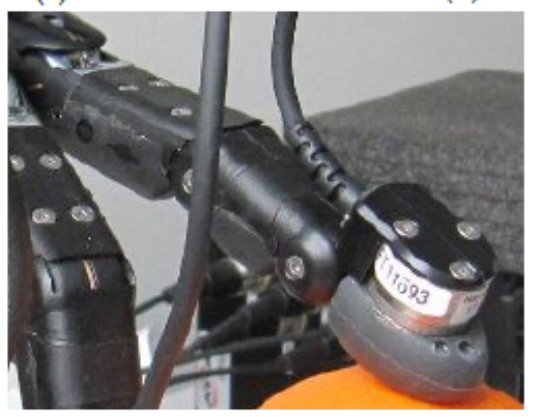

(e)

Figure 11: Advanced integration of tactile sensors on the robot fingertips: (a) a flexible PCB for a capacitive tactile sensing array with 12 taxels designed for the iCub humanoid robot [103],(b) the $\mathrm{iCub}$ flexible PCB wrapped around the inner support of the fingertip [103], (c) a 3D-shaped rigid tactile sensing array with 12 sensing elements attached to the fingertip of the Shadow robot hand [34], (d) the BioTac multimodal tactile sensor installed on the Shadow robot hand by replacing two last links of the finger [43], (e) ATi nano 17 force/torque sensor on the fingertip of the Shadow robot hand [30].

the skin sampling rate for each tactel decreases from 100 $\mathrm{Hz}$ to $25 \mathrm{~Hz}$ as the number of tactels increases. If the real-time pressure distribution is of interest, as for example in tactile servoing [31], the serial data access may fail to produce time-series images of the contact image. The parallel access of data provides higher acquisition rate, but requires a higher number of wires than the serial one. Employing advanced addressing schemes is a way of reducing the number of wires needed in the parallel access schemes. For example, in the row-column scheme [49] $n+m$ wires are needed for $n * m$ array of sensors instead of $n * m+1$ wires required in the schemes with one common ground [34]. Other approaches dedicated to reducing wiring issues include wireless data and power transmission and implementation of a decentralized data pre-processing of tactile signals [24], [76].

\subsection{Integration steps}

One way to integrate tactile sensors in robot hands is in using tactile gloves. A number of tactile data gloves have been designed for use in human grasping applications rather than in autonomous manipulations tasks, e.g. [54]. However, tactile data gloves could be worn on robot hands, 


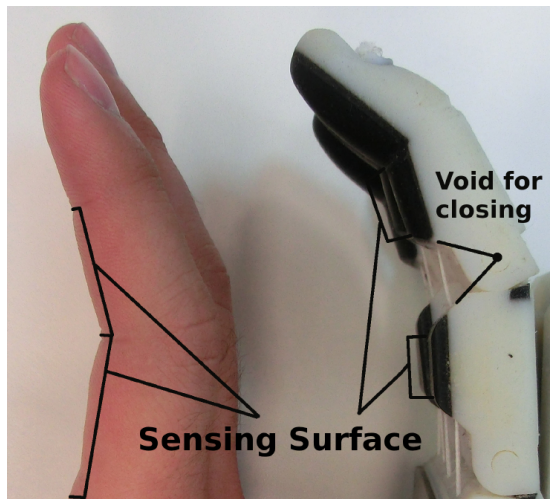

Figure 12: Difference in contact surfaces between a human finger and a robot finger [105].

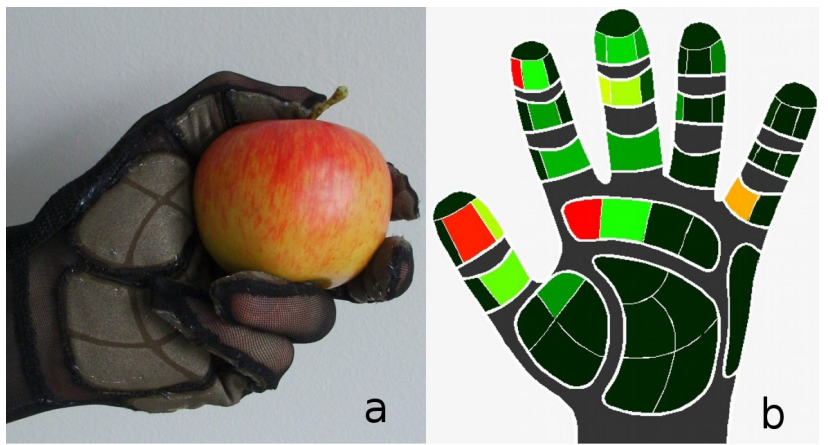

Figure 13: Tactile data glove based on conductive rubber (a) and the tactile information from the data glove during a grasp (b) [54].

as in the Robonaut robot hand [106] capable of sensing 19 points of contact. Commercial tactile data gloves are available from Tekscan [57] and CyberGlove [107]. Figure 13 shows a tactile data glove based on piezoresistive and conductive fabrics.

A more effective way of integrating tactile sensors is to embed them into the robot hand. The embedding procedure of the tactile sensing skin within the robot hand involves the following steps [44]:

- definition of the surface to be covered by the available 3D computer-aided-drawing (CAD) model or by means of a 3D scanner.

- manufacturing of the supporting part using tactile sensing PCBs. This part is to be attached to the robot hand. The use of a $3 \mathrm{D}$ printer can facilitate the manufacturing procedure. This step is not applicable if integration of fingertip-shaped tactile sensors is required, which involves changing the structure of the finger.

- identification and wiring of the sensing elements.

- gluing the sensing elements down on the supporting part.

- covering the sensing elements with flexible material, e.g. silicon rubber. For a specific surface shape, custom molds should be designed.

\subsection{Robot hands equipped with tactile sensors}

This section presents an overview of manipulating platforms with sensorized artificial hands, developed in the framework of research projects in autonomous manipulation and tactile sensing applications. A list of these platforms is presented in Table 3 and a summary with comments about the different hand/sensor combinations are given in Table 4.

In [49] an 8x8 tactile array based on piezoresistive rubber has been attached onto the grippers of the 3-fingered Schunk SDH hand for classifying deformable objects. Outstanding in speed performance, the Lightweight High-Speed Multifingered Hand System [101] integrates Center-of-Pressure (CoP) sensor for the force measurements and PVDF based high sensitive tactile sensor for slip detection, as shown in Figure 14(a) [39]. Commercial 3-finger Schunk SDH hand [109] with integrated Weiss Robotics piezoresistive tactile sensors [58] incorporates a $14 \times 6$ array on each distal link and a 14x7 array on each middle link. The Universal robot hand [35] has 102 tactels on fingertips and 70 tactels on the rest of the links. In contrast to the serial connection of sensors present in the iCub skin [44], each tactile array has its own connection with the acquisition board (Figure 14(b)).

Capacitive arrays from Pressure Profile System [66] have been integrated with PR2 robot grippers [95]. The sensor array on the PR2 robot has tactels in back and front, on left and right sides, and finally on the tip. Very sensitive tactile sensors with fibers connected to capacitive sensor akin to animal whiskers have been integrated with the parallel jaw gripper of a humanoid robot platform to explore object surfaces and for human-robot interaction purposes [113]. The Barret hand [114] has capacitive tactile sensors on the tips, distal link and palm.

The "Takktile" arrays [87] based on barometric measurements have been integrated with the iRobot-HarvardYale (iHY) Hand [42]. The hand is covered by an array of 48 tactels on the palm, a $2 \times 6$ array on proximal links, and a $2 \times 5$ array on distal links with two of the tactels on the tip (Figure 14(c)).

An optical tactile array of 41 tactels with the ability to measure normal and tangential forces has been placed on the tips of a two-fingered robot system [13]. A multimodal tactile sensing system may require a larger space, especially if optical tactile sensors are incorporated within it. Figure 14(d) illustrates the robot hand with the multimodal sensing system [76]. Force torque sensors are placed at the base of each finger, not on the fingertips as in the Shadow hand [104]. The three-axis opto-force sensors [81] can be installed on the tips of the Barret Hand [114].

In [117], photo-reflectors have been attached to the three-fingered robot to provide proximity information for preshaping the fingers during grasping. The Seashell effect sensors [93], which also provide proximity information, have been installed on the PR2 robot grippers.

The tactile sensing system for the DLR robot hand-arm system [116] is designed as large scale tactile skin using the 
Table 3: The list of tactile sensors that have been integrated with robot hands. Number of tactels (No.), spatial resolution (Res.), sensitivity (Sens.), dynamic range (Range) and data acquisition rate (Rate) are provided where possible.

\begin{tabular}{|c|c|c|c|c|}
\hline Tactile sensor & Robot Hand & No. of tactels & Res./Sens./Range & Rate \\
\hline \multicolumn{5}{|c|}{ Piezoresistive sensors } \\
\hline FSR [56] & Robonaut data glove[106] & 19 & $5 \mathrm{~mm} / 0.1 \mathrm{~N} / 20 \mathrm{~N}$ & $1 \mathrm{kHz}$ \\
\hline Fabric sensor [60] & Sensor Glove [54] & 56 & $34 \mathrm{~mm}^{2} /(0.1-30 \mathrm{~N})$ & - \\
\hline Rubber-based [49] & Schunk gripper [108] & $8 \times 8$ & $6.25 \mathrm{~mm}^{2} /-/ 250 \mathrm{kPa}$ & $100 \mathrm{fps}$ \\
\hline Rubber-based [39] & $\begin{array}{c}\text { High-speed 3-fingered } \\
\text { hand [101] }\end{array}$ & $17 \times 19$ & $3 \mathrm{~mm} /-/-$ & $10 \mathrm{kHz}$ \\
\hline Weiss Robotics [53] & Schunk sDH [109] & $(14 \times 6)$ and $(14 \times 7)$ & $3.5 \mathrm{~mm} /-/ 250 \mathrm{kPa}$ & $800 \mathrm{fps}$ \\
\hline 3D-shaped sensor [34] & Shadow Hand & 12 & $5.5 \mathrm{~mm} / 0.03 \frac{\mathrm{N}}{\mathrm{cm}^{2}} / 10 \mathrm{~N}$ & $\sim 1 \mathrm{kHz}$ \\
\hline Rubber-based [35] & Universal robot hand [35] & 102 on tip & $3.6 \mathrm{~mm} / 1 \mathrm{~N} /-$ & $50 \mathrm{~Hz}$ \\
\hline Gifu hand sensor & Gifu Hand III [110] & 624 & $\sim 4 \mathrm{~mm} /-/ 22 \frac{N}{\mathrm{~cm}^{2}}$ & $10 \mathrm{~Hz}$ \\
\hline Tekscan [57] & Shadow Hand [94] & 349 & $4 \mathrm{~mm} /-/ 345 \mathrm{kPa}$ & $200 \mathrm{~Hz}$ \\
\hline FSR [41] & Southampton hand [41] & 15 & - & - \\
\hline ATi Nano17 sensors [61] & Shadow hand [98] & 5 per finger & $-/ 3.26 \mathrm{mN} / 12 \mathrm{~N}$ & $833 \mathrm{~Hz}$ \\
\hline Weiss Robotics [58] & Fluidic FRH-4 hand [111] & $14 \times 6$ & $3.5 \mathrm{~mm} /-/ 250 \mathrm{kPa}$ & $230 \mathrm{fps}$ \\
\hline \multicolumn{5}{|c|}{ Capacitive sensors } \\
\hline Icub sensor [103], [44] & iCub Humanoid robot & 12 per tip, 48 -palm & $7 \mathrm{~mm} / 2.5 \frac{\mathrm{fF}}{k P a} / 150 \mathrm{kPa}$ & $25-250 \mathrm{~Hz}$ \\
\hline PPS sensors [66] & PR2 robot grippers [38] & 22 & $4 m m / 6.25 \mathrm{mN} / 7 \mathrm{kPa}$ & $24.4 \mathrm{~Hz}$ \\
\hline PPS RoboTouch [66] & Allegro robotic hand [64] & 24 & $25 \mathrm{~mm}^{2} / 7 \mathrm{kPa}$ & $30-100 \mathrm{~Hz}$ \\
\hline Dynamic sensor [9] & Robotiq Gripper [112] & 132 & $-/-12 \mathrm{~N}$ & $300 \mathrm{~Hz}$ \\
\hline Combined sensor [113] & Parallel jaw gripper [113] & 16 & $10 \mathrm{mN}$ & $\begin{array}{l}\text { up to } 35 \\
\mathrm{kHz}\end{array}$ \\
\hline PPS RoboTouch & Barrett Hand [114] & 120 per finger & $5 \mathrm{~mm} / 6.25 \mathrm{mN} / 7 \mathrm{kPa}$ & $30-100 \mathrm{~Hz}$ \\
\hline \multicolumn{5}{|c|}{ Piezoelectric sensors } \\
\hline PRes. [58] + PVDF [24] & 8 DoF Fluid Hand [70] & $4 \mathrm{x} 7$ & $3.5 \mathrm{~mm} /-/ 250 \mathrm{kPa}$ & $\geq 1 \mathrm{kHz}$ \\
\hline PRes. ink + PVDF [88] & SKKU Hand II [88] & 24 on fingertip & $0.5 \mathrm{~mm} /-/-$ & - \\
\hline Tactile skin [115] & DLR Hand [116] & in & process of & development \\
\hline \multicolumn{5}{|c|}{ Barometric measurements based sensor } \\
\hline Takktile (silicon) [87] & iHY Robot Hand [42] & $24+48$ & $5 \mathrm{~mm} / 10 \mathrm{mN} / 4.9 \mathrm{~N}$ & $50 \mathrm{~Hz}$ \\
\hline BioTac (liquid) [83] & Shadow Hand [98] & 1 per finger & $-/ 0.1 \mathrm{~N} / 3 \mathrm{~N}$ & $1 \mathrm{kHz}$ \\
\hline \multicolumn{5}{|c|}{ QTC tactile sensors } \\
\hline Robonoaut sensors & Robonaut Hand [75] & 33 & $-/ 0.1 \mathrm{~N} / 10 \mathrm{~N}$ & - \\
\hline Piratech [73] & Shadow Hand [74] & 36 & $3 \frac{k N}{m^{2}} / 400 \frac{k N}{m^{2}}$ & - \\
\hline \multicolumn{5}{|c|}{ Optical tactile sensors } \\
\hline Sensor for MRT [77] & Robot Manipulator & 9 & $-/ 0.5 \mathrm{~N} / 5 \mathrm{~N}$ & $25 \mathrm{fps}$ \\
\hline 3 DoF sensor [37] & Robot gripper & 41 & $3 \mathrm{~mm} / 0.08 \mathrm{~N} / 1.8 \mathrm{~N}$ & $10 \mathrm{~Hz}$ \\
\hline Optoforce [81] & Barret Hand & 1 per finger & $10 \mathrm{~mm} /-/ 10 \mathrm{~N}$ & - \\
\hline \multicolumn{5}{|c|}{ Multi-modal tactile sensors } \\
\hline Proximity Sensor [90] & A three-fingered hand [90] & Palm: $5 \times 6$ & $10 \mathrm{~cm}$ and $2 \mathrm{~cm}$ & $1 \mathrm{kHz}$ \\
\hline BioTac sensor [84] & Shadow Hand [43] & $\begin{array}{c}19+\text { fluid } \\
\text { barometer }+ \\
\text { thermistor }\end{array}$ & $-/ \sim 0.01 \mathrm{~N} / 1: 1000$ & $\begin{array}{c}50 \mathrm{~Hz} ; 2 \mathrm{kHz} \\
50 \mathrm{~Hz}\end{array}$ \\
\hline Optical + PVDF + Force & 3-fingered gripper $[76]$ & $\begin{array}{l}324 \text { fibers, } 120 \\
\text { PVDF }, 3 \mathrm{~F} / \mathrm{T}\end{array}$ & $-/-/ 4 \mathrm{~N}$ & $\begin{array}{l}30 \mathrm{fps} ; \\
10 \mathrm{kHz} ; \\
100 \mathrm{~Hz}\end{array}$ \\
\hline \multicolumn{5}{|c|}{ "Structure-borne sound" tactile sensors } \\
\hline Microphone & Oxford prosthesis [32] & 1 & - & - \\
\hline Accelerometer & PR2 robot grippers [38] & 1 & $0.15 \mathrm{~m} / \mathrm{s}^{2}$ & $3 \mathrm{kHz}$ \\
\hline SeaShell effect sensor [93] & PR2 robot grippers & 1 & -/Non/Non & $44 \mathrm{kHz}$ \\
\hline
\end{tabular}




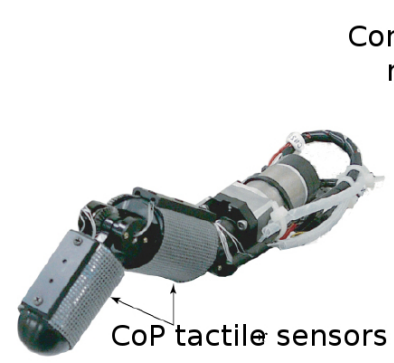

(a)

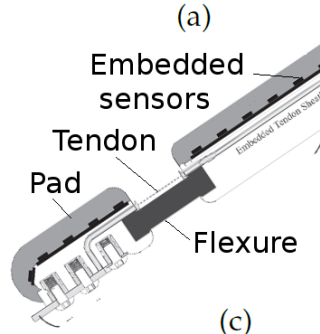

(c)

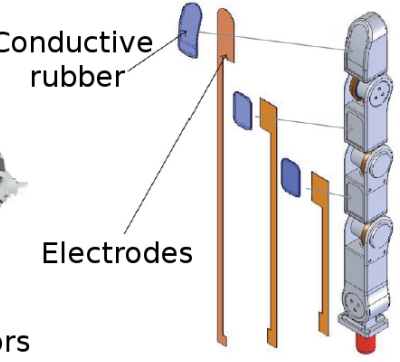

(b)

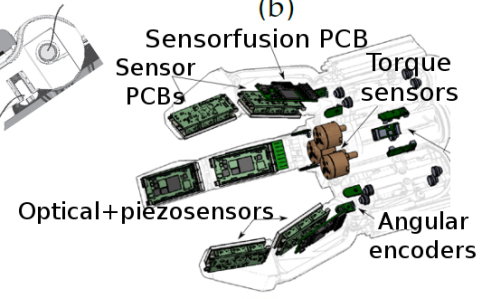

(d)
Figure 14: Three-fingered robot hands with tactile sensors: (a) a finger with tactile sensor of the 3-fingered high-speed robot hand [101], (b) assembly of tactile sensing arrays with a robot finger of the Universal robot hand with 3 movable and 2 immovable fingers [35], (c) schematic illustration of a finger of the iHY robot hand with embedded array of pressure sensors based on digital barometers placed inside the soft paddings of the fingers [42]; (d) schematic illustration of the integration of a multimodal sensing system with a three-fingered robot hand [76]

column-row net structure [115]. The robonaut hand has tactile feedback through the tactile data glove incorporating piezoresistive technology [106] and QTC technology [75]. Figure 15(a) shows the Fluidic hand [70] with modified version of the Weiss [58] sensors. The dexterous Gifu III robot hand (Figure 15(d)) has a sensing array of 859 taxels (Figure 15(c)) based on piezoresistive conductive ink [110]. An array of 24 conductive ink in combination with piezoelectric PVDF material has been used in the SKKU II robot hand [88]. The Shadow Hand [98] has different integrated tactile sensors: force/torque sensors (Figure 11(e)) [30], multimodal Biotac tactile sensors (Figure 11(d)) [43], 3D-shaped fingertip tactile sensors (Figure 11(c)) [34], and QTC sensors [74]. The robot hand of the iCub humanoid robot [100] has sensors on the fingertips and palm, but not on the middle and proximal phalanges (Figure 15(b).

Besides the five fingered robot hands, a number of anthropomorphic robot hands with three fingers and thumb exist, including the "Twendy one" robot hand covered by capacitive tactile sensing arrays [118] and the "Allegro" robot hand [119] developed by SimLab Co.

\subsection{Large area tactile skin}

There is a high demand for manipulators and humanoid robots whose whole surface is covered with tactile sensors [18]. Large sensing areas embedded in robotic systems enhance human-robot interaction and are important for safety reasons. However, a large area tactile skin and the
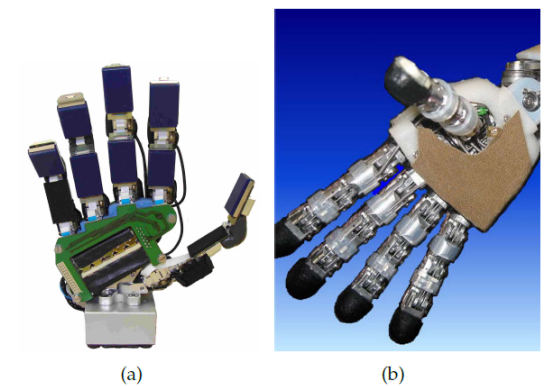

(b)

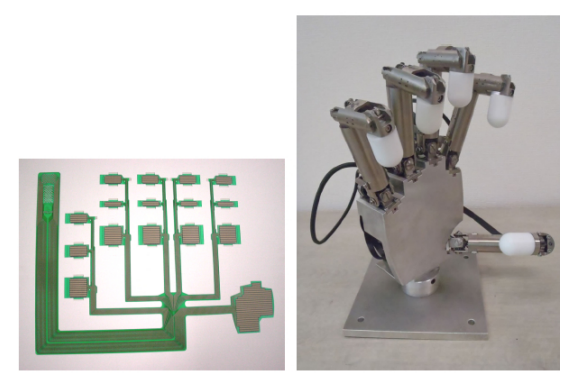

(c)

(d)

Figure 15: Five-fingered robot hands with tactile sensors: (a) the fluidic robot hand with combined piezoelectric and piezoresistive tactile sensors that can sense high-frequency vibrations due to the absence of electric motors [24], (b) the robot hand of the iCub humanoid robot with tactile sensors on the fingertips and the palm [44], (c) flexible tactile sensing arrays of the SKKU robot hand [88], (d) the SKKU robot hand [88]

concomitant increase in the amount of tactels present challenges with regards to optimal data acquisition and wiring.

The number of sensing tactels should be easily changeable for arbitrary surfaces to enhance the performance of the system. The iCub skin uses flexible triangle patches consisting of 12 sensing tactels each and off-the-shelf CDC AD7147 [67] (Figure 2(c)). Up to 16 triangle patches in series can be connected with each other but only one of them must be connected with the micro-processing unit which significantly reduces the amount of wires required. However, polling time increases proportionally to the number of serial sensing elements. The iCub skin has been integrated in the child-sized humanoid robot KASPAR [120] and the autonomous humanoid robot NAO. iCub skin based on capacitive technology can sense applied pressure only.

Unlike the capacitive technology based iCub skin which can only sense applied pressure, HEX-O-SKIN measures temperature, vibrations and light touch [121]. Each patch of the HEX-O-SKIN is a hexagonal printed circuit board equipped with proximity sensors, accelerometers, thermistors, and a local controller. Each patch is less than $2 \mathrm{~g}$ in weight, $5.1 \mathrm{~cm}^{2}$ in area, and $3.6 \mathrm{~mm}$ thick.

A limited number of tactile sensing skins has been integrated in robotic manipulators for applications that require tactile feedback, as in safe human-robot interaction. An example of an industrial manipulator covered with an array of capacitative proximity sensors is described in 
Table 4: Sensors integrated with robot hands: advantages and disadvantages of major approaches.

\begin{tabular}{|c|c|c|}
\hline Hand/Sensor Combination & Advantages & Disadvantages \\
\hline $\begin{array}{c}\text { 3D-shaped array [34] \& Shadow } \\
\text { Hand; iCub robot fingertip } \\
\text { sensor [68] }\end{array}$ & $\begin{array}{c}\text { Multiple point of contact, covers } \\
\text { spherical shapes, wires - within } \\
\text { fingers }\end{array}$ & $\begin{array}{l}\text { Normal force measurements } \\
\text { only }\end{array}$ \\
\hline $\begin{array}{c}\text { Ellipsoid f/t sensor [104] \& } \\
\text { Shadow Hand; OptoForce [81] \& } \\
\text { Barret Hand }\end{array}$ & $\begin{array}{l}\text { Covers spherical shapes, high } \\
\text { sensitivity, shear forces. }\end{array}$ & $\begin{array}{l}\text { Single point of contact only, } \\
\text { wires - outside of fingers }\end{array}$ \\
\hline BioTac [84] \& Shadow Hand & $\begin{array}{l}\text { Multiple point of contact, high } \\
\text { bandwidth, wires - inside }\end{array}$ & Last joint static (20 degrees) \\
\hline Robonaut glove and Hand [106] & Ease of replacement, low cost & $\begin{array}{l}\text { Not reliable compared to rigidly } \\
\text { attached sensors }\end{array}$ \\
\hline Fabric sensor [54] & Ease of replacement, stretchable & Wear and tear off \\
\hline $\begin{array}{c}\text { Tactile sensing array (PPS [66], } \\
\text { Tekscan [57], and etc.) \& any } \\
\text { robot hand }\end{array}$ & $\begin{array}{l}\text { Can be easily attached to any } \\
\text { flat and cylindrical surfaces }\end{array}$ & $\begin{array}{c}\text { Cannot cover spherical shape, } \\
\text { wiring issues }\end{array}$ \\
\hline $\begin{array}{l}\text { Weiss Robotics [58] \& any robot } \\
\text { hand; Takktile [87] \& iHY hand }\end{array}$ & Robust & Flat surface only \\
\hline $\begin{array}{l}\text { SeaShell effect sensor (Cavity } \\
\text { with microphone \& PR2) [93] }\end{array}$ & Pre-touch sense & $\begin{array}{l}\text { Direct contact of the cavity with } \\
\text { an object limits forces }\end{array}$ \\
\hline Proximity Sensor [90] & Pre-grasp sense & $\begin{array}{c}\text { Cannot measure very close } \\
\text { proximities }\end{array}$ \\
\hline $\begin{array}{l}\text { Accelerometer at the base of } \\
\text { robot grippers [38] }\end{array}$ & Vibration detection & $\begin{array}{c}\text { Interference with electric motor } \\
\text { noise }\end{array}$ \\
\hline $\begin{array}{l}\text { Microphone at the tips of the } \\
\text { Oxford Hand prosthesis [32] }\end{array}$ & Vibration detection & $\begin{array}{l}\text { No interference with motor } \\
\text { noises }\end{array}$ \\
\hline
\end{tabular}

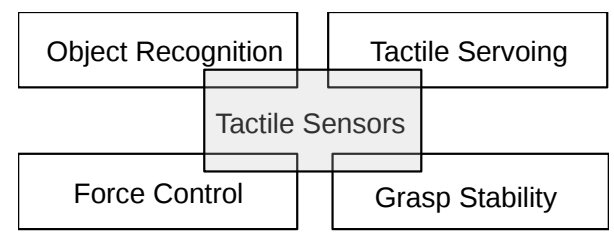

Figure 16: Tactile sensing techniques. Tactile sensing in robot hands is used for object recognition, tactile servoing, force control and for assessing grasp stability.

[21]. A commercial industrial manipulator that incorporates 118 proximity sensors is shown in [122]. Research in design of multi-fingered dexterous robot hands, being previously focused on prosthetic hands only, has surged in recent years. Various dexterous robot hands were developed in research laboratories and became commercially available [74], [118], [119].

\section{Computational Techniques in Tactile Sensing Ap- plications}

In the robotics literature, tactile feedback has been widely used for telemanipulation, haptic devices, and legged robots [123]. In event-driven manipulation, tactile signals have been used for detection of the current manipulation phase (contact/no contact, rolling, sliding) [124].
The use of tactile information for object exploration and recognition, material classification, and slip prediction has recently become rather popular as is reflected in [40], [95], [23], [19].

In robot hand applications, tactile signals are used to recognize objects, control forces, grasp objects, and to servo surfaces (Figure 16). Each of these applications will be discussed in following sections. The major computational techniques used in these applications are illustrated in (Figure 17). As discussed in the Section 2.2, different tactile sensor types have different sensing quantities, including force vectors, vibrations, and contact patterns. These quantities are then subjected to various computational techniques. The same computational technique may be used in a number of applications, as it illustrated in the latter figure.

\subsection{Grasp stability and slip detection}

Grasping is one of the basic skills service robots and industrial manipulators are expected to have. Before performing a grasping procedure, a robot must plan the grasp. Grasping is a complex process for robot hands even if object parameters such as shape, position, physical properties are known. When the properties are known, analytical approaches involving force and form closures can be employed to perform grasping [125]. In unstructured environ- 


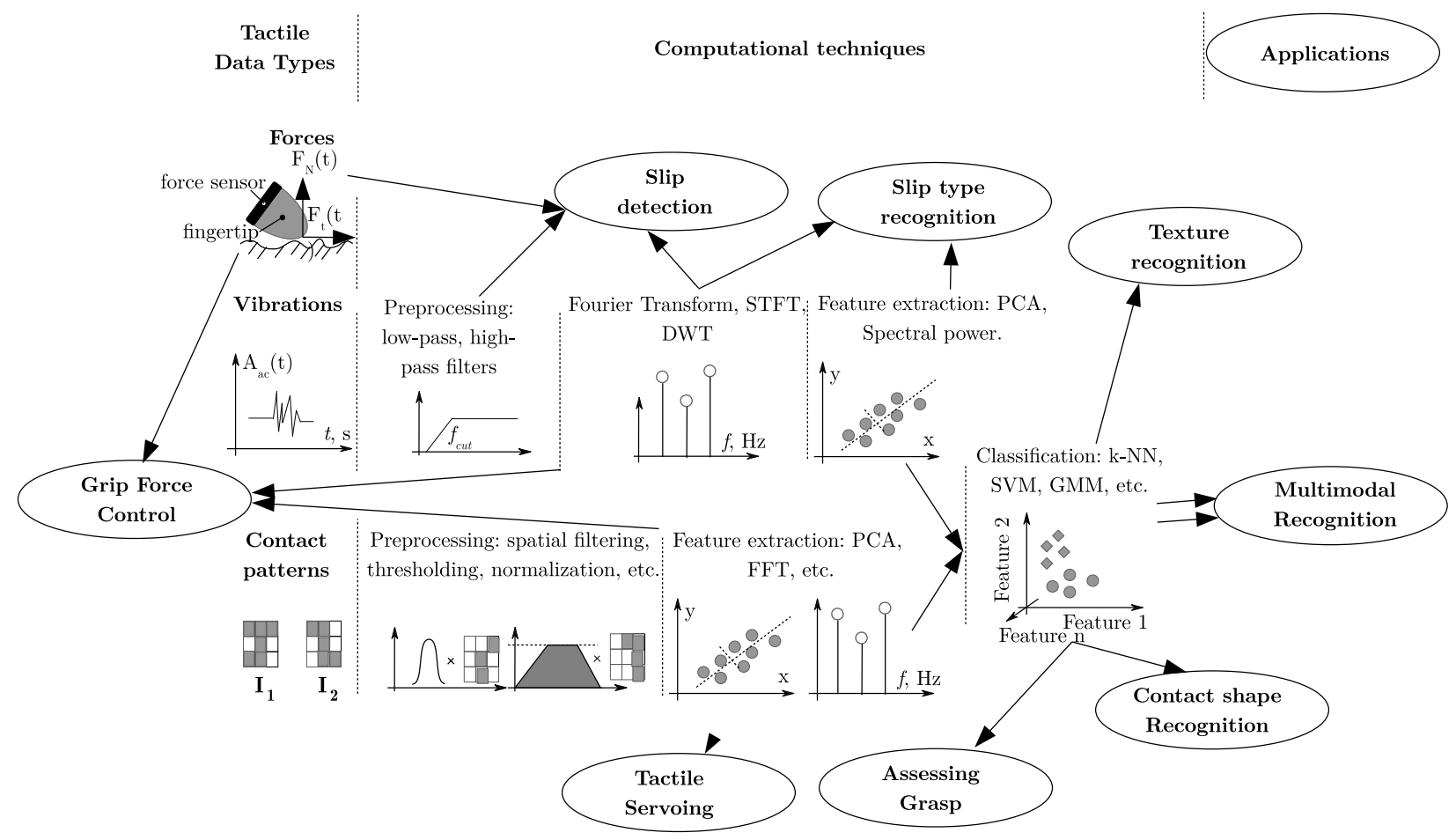

Figure 17: Overview of computational techniques applied to tactile sensing signals in the reviewed robot hand applications. Each tactile data type is shown on the left. The computational techniques applied to the tactile signal are shown in the middle. Different applications of sensorized robot hands exploiting these techniques are shown on orbital on oval blocks. Arrows indicate only the major techniques of deriving information.

ments, object parameters are uncertain, which makes the grasping task even more difficult and presents a big challenge for grasp stability approaches. A detailed review of all grasping techniques is out the scope of this paper and can be found in previous papers [126], [127].

In some approaches, the robot grasping procedure could be simplified by using proximity sensors on fingertips [128]. There are two main approaches of robot grasping that involve tactile feedback. One approach treats grasping as a control problem and does not consider hand kinematics or assumes simple hands like grippers [39]. Another approach makes use of both model based grasp planning and force feedback to address the problem of grasping with dexterous robot hands that have more dof than grippers [25].

Regarding tactile sensor types and the way of processing the data, there are three different techniques for assessing grasp stability at the current state-of-the-art: friction cone based techniques, vibrations based techniques, and tactile images based techniques. Each of the technique is discussed in following.

\subsubsection{Friction cone estimation for the slip event}

The friction coefficient of surfaces and the load conditions are very important in grasping. When humans pick up an object, they take into account these parameters and adjust grasping forces based on tactile feedback during manipulation. The stability of a grasp is evalu- ated by the ratio of normal, $F_{n o r m}$, to tangential, $F_{t a n g}$, reaction forces and static coefficient of friction $\mu_{f}$ (Figure 18(a)). Maintaining objects within the friction cone, to preclude slippage, is ensured by the following condition [125]: $1<\mu_{f} \times \frac{F_{\text {norm }}}{F_{\text {tang }}}$. The tangential force can be obtained by force/torque $(\mathrm{F} / \mathrm{T})$ sensors, for example ATi Nano 17 [61], whereas most of the current pressure sensing arrays can measure normal pressure only (Figure $8(\mathrm{c})$ ). In [86] tangential forces are computed by applying a Kalman filter to the data of the pressure sensing arrays of a bio-mimetic tactile sensor. The sensor consists of conductive fluid and electrodes placed in different places of the fingertip. Hence, the sensor does not provide absolute force values. The Kalman filter integrates signals from the electrodes to produce a force output. Other approaches can rely on dynamic friction models that allow the prediction of an incipient slip. For example, using $\mathrm{F} / \mathrm{T}$ sensors installed on the Barret hand [114], Song et al. [27] estimate the coefficients of the dynamic LuGre friction model of a contact with an unknown object through two exploratory motions. Break-away friction ratio (BF-ratio) is then computed to predict a slippage. Besides the transduction methods mentioned in Section 2.1, heat microflux detectors, which are mainly used for measuring objects' thermal properties, can be used for detecting a slip [129]. The temperature at the contact point increases during the slip due to the energy dissipation at the presence of friction 
forces.

\subsubsection{Vibrations as the slip-signals}

Except exploratory procedures such as texture recognition, the key feature of a stable grasp is the absence of slippage [43]. During slippage or at the moment of contact with the environment, a robot hand experiences mechanical vibrations. This phenomenon is known as structureborne sound [130]. The absence of vibration frequencies indicates the absence of slippage. Achieving stable grasp by detecting vibrations has been long implemented in hand prosthetic devices [16], [131], [132]. In order to detect vibrations during a slip event, the tactile sensor should have appropriate bandwidth to detect the vibration frequencies (Section 2). Piezoelectric materials (Figure 3) and capacitive sensors (Figure 2) have been widely used for detecting vibrations induced by a slip. These sensors are usually embedded into pressure sensitive tactile arrays. Signals coming from each sensor represent high-frequency oscillations (Figure 8(b)) and are sampled at a high sampling rate.

Dynamic tactile signals can be processed directly in time domain and in frequency domain. One of the simplest ways of detecting the slippage is to use a high-pass filter (Figure 18(b)). A given level of filtered disturbances indicates a slip-event. In [38] forces of each cell in capacitive tactile sensing array are subjected to a discrete-time firstorder Butterworth high-pass filter with cut-off frequency of $5 \mathrm{~Hz}$ to mimic fast adaptive (FA-II) human afferents. A high-bandwidth accelerometer is used to detect contact between the object and the environment. The detection of slippage by evaluating the level of high-passed filtered data can be processed at a high rate.

Another computational technique using vibrations is based on the transformation to the frequency domain and the calculation of the spectrum power, as shown in Figure 18(c). In [39] pressure disturbance signals are subjected to discrete wavelet transform (DWT) [134]. When DWT power exceeds the experimentally determined threshold, initial slip is detected and the grasping force is increased accordingly. Cutkosky et al. [9] developed a technique to distinguish between two types of slippage: robot hand / object and object / environment. Acquired data from these two types of slippage were identified by the parameter noted as power-ratio classifier, which is calculated by applying Fourier transformation and phase shifting in frequency domain. The power-ratio classifier is the ratio of the spectrum power of the individual tactel to the power spectrum of all tactels. Tactile signals are processed in a way that mimics the effects of stimuli on human tactile receptors, both individually and as an ensemble. Slip is classified by values of relative power between individual tactels and the array as an ensemble.

A further computational technique uses transformation to frequency domain and then applies principal component analysis (PCA) and machine learning methods (Figure $18(\mathrm{~d}))$. In [24], input signal $(x[n])$ is processed by the Short-Time Fourier Transformation (STFT) with window function in a short period of time $(w[n])$, which provides a two dimensional representation in time-frequency domain: $\operatorname{STFT}\{x[n]\} \equiv X(m, w)=\sum_{n=-\infty}^{\infty} x[n] w[n-m] e^{-i w n}$. The transformed signal is then subjected to PCA and the slip is detected by k-NN (k nearest neighbor) classifier.

Depending on the transduction type of the sensor, a stable grasp can be qualitatively assessed from: (1) contact forces [27], (2) vibrations [9], and (3) tactile contact patterns and hand kinematics [25]. Figure 18 outlines different algorithms and computational techniques that have been used for achieving and assessing the grasp. Table 5 lists the robot hands and tactile sensors that have been tested with the above techniques.

\subsubsection{Tactile image features for stable grasp estimation}

Data from tactile sensing arrays can be treated as a gray scale image (Figure $8(\mathrm{a})$ ). When an object comes to contact with the tactile array, tactile image features of the contact pattern can be extracted for the further estimation of a stability of a grasp.

The first technique introduced in [133] detects the slippage of an object by analyzing changes of feature points of the tactile image. Data is collected at a sampling rate of $60 \mathrm{~Hz}$ from a $44 \times 44$ array of piezoelectric sensors installed on an industrial manipulator. Before the actual motion of the grasped object in a slip-event, there are some feature points that remain on previous positions and points that have moved. Ratio of immobile points to moved points indicates the slip-event. This approach requires a large tactile array because the surface of an object that is in contact should be fully represented in the tactile image.

The slip detection techniques demonstrated in the previous sections can be used in grasping approaches that address the grasp as a control problem and do not take into account the hand kinematics. For the dexterous robot hands with tactile sensing arrays, a grasp stability can be estimated by computing tactile information together with hand kinematics (Figure 18(e)).

Bekiroglu et al. [95] consider grasp stability as a probability distribution that depends on tactile images acquired from pressure distribution sensing arrays; joint configuration of the hand; object information (e.g. object shape class) and grasp information (e.g. hand pre-shape). Grasp stability is evaluated by analyzing tactile images and hand configurations based on supervised machine learning algorithms. While AdaBoost [135] and Support Vector Machine (SVM) [136] classifiers are used for one-shot recognition at the final step of the grasping procedure, the hidden Markov model (HMM) [137] classifier is used for the time-series case. It should be noted that, besides the SVM, Adaboost and kNN classifying algorithms, other classification, clustering, statistical learning and data mining algorithms described in [138] can be used for the grasp stability estimation. Dang et al. [25] developed a grasping framework that generates grasps; executes and then estimates the quality of the grasp and performs hand adjustment and local geometry exploration if the grasp is not successful. 


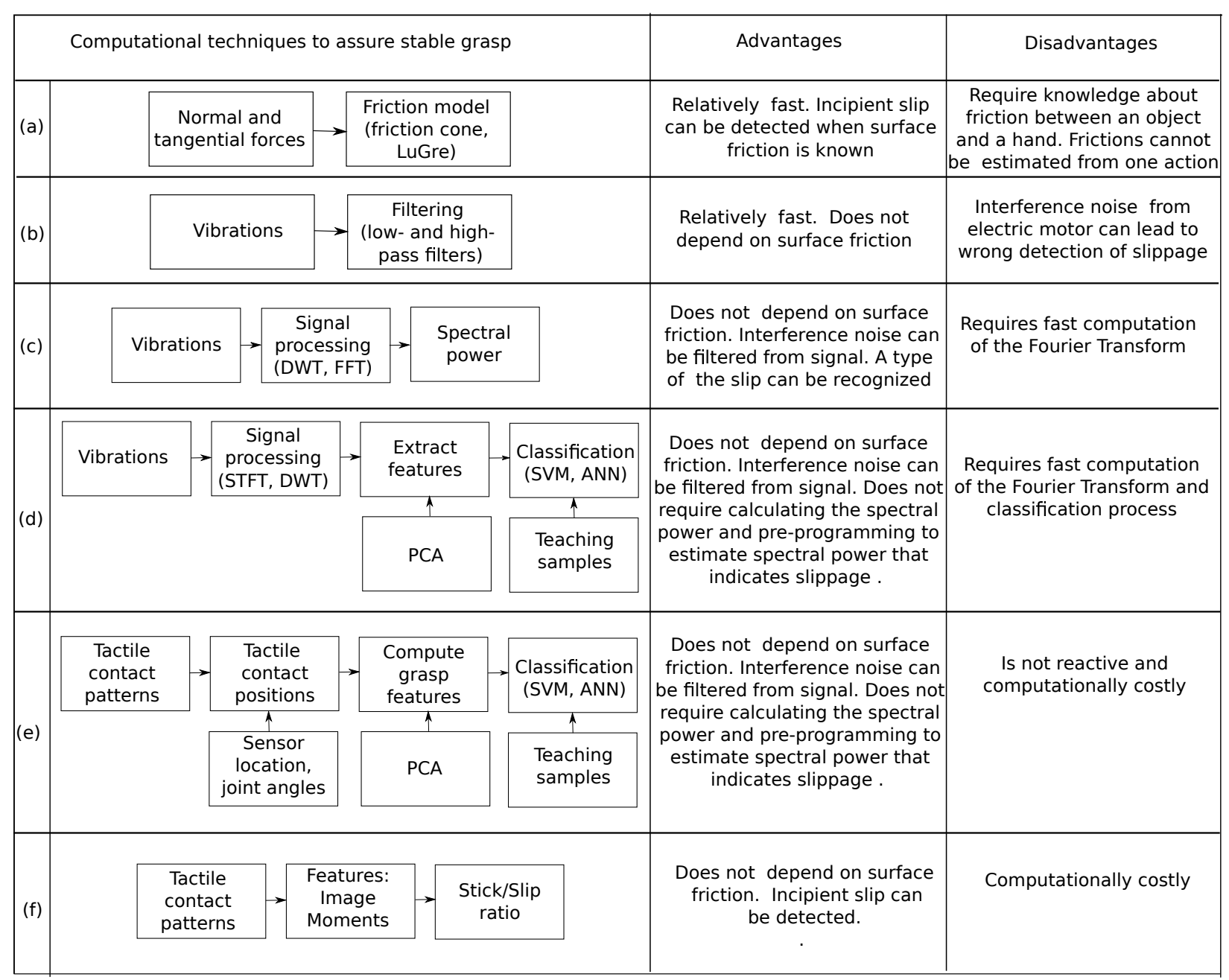

Figure 18: Data processing steps for the techniques applied in tactile-based stable grasping: (a) slip detection based on static (e.g. friction cone) and dynamic (e.g. LuGre) contact force models [27]; (b) slip detection based on vibrations that can be recognized in time domain by existence of high-pass filtered tactile data [38]; (c) slip detection based on vibrations by calculating a spectral power in the frequency (i.e. Fourier transformations) and time-frequency (i.e. wavelet transformations) domains [39], [9]; (d) slip detection based on vibrations that can be recognized in time-frequency domain by extracting and classifying features of transformed signals [24]; (e) grasp stability estimation based on features from tactile images and hand kinematics [95], [25].

Grasp stability is estimated from tactile images. Unlike to the algorithm of Bekiroglu et al. [95], the position of each tactile array is calculated to determine the configuration of the contacts involved in a grasp. Then grasp feature vectors are computed using bag-of-words model and classified by a supervised SVM classifier. If a grasp is not successful, the robot adjusts the hand according to tactile experience database of stable grasps or explore the local geometry.

Other rather old approach proposed by Kyberd et al. [41] detects slippage by calculating changes in tactile pattern represented by a matrix in which the increase of force corresponds to 1 , decrease to $(-1)$, and no changes to $(0)$. Slippage and twist are derived then by summing and subtracting the neighbor elements in the matrix.

The advantages and disadvantages of the above approaches are given in the Figure 18. In the case of estimation of grasp stability by measuring normal forces, the friction surface must be given in advance or estimated by tangential force measurements (Figure 18 (a)). Meanwhile, the rest approaches do not require this preliminary information about surface. However, the second approach of detecting vibrations by applying a high-pass filter (Figure 18 (b)) may suffer from an interference noise coming from electric motors. This interference can be eliminated by transforming temporal signals to the frequency domain and filtering out motor noise harmonics ((Figure 18 (b)). These approaches are well suited for reactive controllers. But in grasp planning algorithms, information about contact patterns play an essential role.

Regarding the sensor parameters, high temporal resolution is very important for the vibration based techniques and less important for the one based on friction cone estimation. High spatial resolution increases performance of the approach of assessing grasp stability from contact pat- 
Table 5: Approaches for the grasp stability estimation based on tactile information. A number of computational techniques for assessing the grasp based on vibration, friction force model and tactile images are listed in accordance with used tactile sensors and robot hands.

\begin{tabular}{|c|c|c|c|}
\hline Sensors & Robot Hands & Techniques & Ref. \\
\hline \multicolumn{4}{|c|}{ Vibrations } \\
\hline Weiss Robotics + PVDF & Fluidic Hand [70] & Filtering, STFT, PCA, kNN & {$[24]$} \\
\hline PPS sensors & PR2 gripper & Filtering, Grip force control & {$[38]$} \\
\hline $\mathrm{CoP}+\mathrm{PVDF}[39]$ & High-speed hand hand & DWT power, force control & {$[39]$} \\
\hline Capacitive sensors & Robotiq gripper [112] & $\begin{array}{c}\text { FFT, spectral power, phase shift, slip type } \\
\text { detection }\end{array}$ & {$[9]$} \\
\hline Microphone & Prosthetic hand & Filtering & {$[32]$} \\
\hline Accelerometer & $\mathrm{PR} 2$ robot grippers & Filtering, object - world contact detection & [38] \\
\hline \multicolumn{4}{|c|}{ Friction force model } \\
\hline BioTac $[86]$ & Otto Bock M2 hand & Force control, Kalman filter & {$[86]$} \\
\hline ATi nano 17 [61] & Barret hand & $\begin{array}{l}\text { LuGre dynamic friction model, break-away } \\
\text { ratio }\end{array}$ & {$[27]$} \\
\hline \multicolumn{4}{|c|}{ Tactile images } \\
\hline Piezoresistive [58] & $\begin{array}{l}\text { Schunk 3-Finger Gripping } \\
\text { Hand sDH [109] }\end{array}$ & $\begin{array}{c}\text { Temporal and Static image features }+ \text { joint } \\
\text { angles, HMM and SVM }\end{array}$ & {$[95]$} \\
\hline Capacitive sensor arrays & Barret hand & Image features + joint angles, SVM & {$[25]$} \\
\hline Piezoelectric $(16 \times 16)$ & Manipulator & $\begin{array}{l}\text { Image moments, Localized Displacement } \\
\text { Phenomenon, incipient slip }\end{array}$ & [133] \\
\hline
\end{tabular}

terns. And high sensitivity is essential for the techniques that rely on estimation of surface friction.

\subsection{Tactile Object Recognition}

Object recognition is an important element in humanrobot interaction and autonomous manipulation [150]. In manipulation tasks, robotic systems detect, explore and recognize objects. For the detection and recognition tasks robots use their perception system. The perception system includes audio, vision and tactile subsystems. Information from audio devices - high sensitive microphones that can detect micro-vibrations - serves to detect a slip [32] and to recognize textures [151]. Visual information is provided by RGB cameras, stereo cameras, RGB-Depth cameras, laser scanners, and etc. Image information can be sufficient to control a robot in some applications as in visual servoing. However, recent trends show that, even though robotic vision gives a lot of information, tactile information about the contact is still necessary as it improves performance of the recognition and manipulation tasks [17]. Data from vision may be noisy or even not available when the robot itself obstructs visibility during manipulation. Tactile information from end-effector can complement the information acquired from vision for object detection and recognition. Tactile sensors can provide information about local surface texture, as for example in [79].

Depending on the sensor type, there are three different approaches of tactile object recognition (Figure 19). The first approach of object identification, a robot hand uses multimodal tactile information [43]. Different tactile signals are combined to identify an object in contact with the

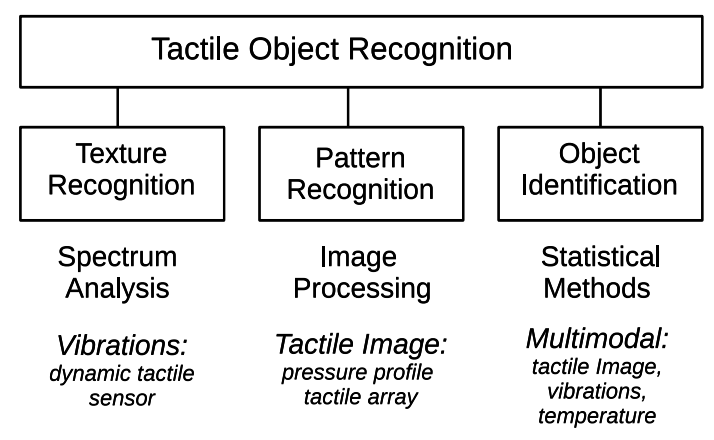

Figure 19: Tactile object recognition. Tactile data can be used for classification of textures based on spectrum of frequencies that appear during the sliding over a surface [43]. Object that is in contact with the tactile sensing array can be recognized from the contact patterns using image processing techniques [40]. When multimodal tactile information is available, objects can be identified through statistical and probabilistic analysis of multimodal data [19].

sensors. The second approach is based on spectral analysis. The texture of a surface is identified via vibrations which occur when a tactile sensor slides over the surface. Oscillations are transformed to frequency (time-frequency) domain to detect a different texture according to the spectrum of the acquired signal [24]. In the last approach of the contact pattern recognition, image processing techniques are applied in order to recognize the shape of the object that is in contact with a sensing array [40]. Tactile images can be also used to classify deformable and rigid objects [49] and in some specific cases for texture recognition [79] from a contact print with high resolution. 
Table 6: Tactile Object Recognition. A number of computational techniques for object identification, texture classification and contact pattern recognition is listed in accordance with tactile sensor types and robot hands.

\begin{tabular}{|c|c|c|c|}
\hline Tactile Sensors & Hands & Methods & Ref. \\
\hline \multicolumn{4}{|c|}{ Object Identification } \\
\hline $\begin{array}{l}8 \mathrm{x} 8 \text { array and } 6 \mathrm{x} 14 \text { Weiss } \\
\text { sensor }\end{array}$ & $\begin{array}{c}\text { Schunk Gripper and SDH } \\
\text { hand }\end{array}$ & Image Moments, k-NN, DTW & {$[49]$} \\
\hline BioTac [84] & Shadow Hand & ANN, GMMR, PCA & {$[43]$} \\
\hline $\begin{array}{c}\text { Capacitive } \\
\text { array }+ \text { microphone }\end{array}$ & Barret Hand & $\begin{array}{c}\text { Multimodal categorization using statistical } \\
\text { model }\end{array}$ & [139] \\
\hline \multicolumn{4}{|c|}{ Texture Recognition } \\
\hline BioTac sensor [84] & Shadow Hand & FFT, SVM, Bayesian approach & [19] \\
\hline Ati Nano 17 & Barret hand & friction ratio, $\mathrm{FFT}, \mathrm{k}-\mathrm{NN}$ & [140] \\
\hline Digital accelerometer & - & STFT, k-NN, SVM & [141] \\
\hline PVDF based & $\begin{array}{l}\text { Artificial robotic finger } \\
\text { from Robotis motors }\end{array}$ & $\begin{array}{c}\text { FFT, majority voting, naive Bayes tree } \\
\text { (NBTree), naive Bayes, decision trees (J48) }\end{array}$ & {$[142]$} \\
\hline Accelerometer & - & $\begin{array}{c}\text { Feature extraction, SVM, Pitman-Yor } \\
\text { process mixture models }\end{array}$ & {$[143]$} \\
\hline GelSight & - & MSLBP & {$[79]$} \\
\hline \multicolumn{4}{|c|}{ Contact Pattern and Shape Recognition } \\
\hline PVDF + conductive foam & Fluid Hand [70] & PCA, Moments, k-NN & {$[24]$} \\
\hline PPS [66] & - & $\begin{array}{l}\text { RRT, k-means, GMMs, Bag-of-Features, } \\
\text { PCA, SIFT, MR-8, Polar Fourier }\end{array}$ & {$[40]$} \\
\hline Joystick sensor & 6 DoF Manipulator & $\begin{array}{l}\text { Curvature estimation, surface normal, } \\
\text { model-based recognition }\end{array}$ & [144] \\
\hline Piezoresistive rubber & $\begin{array}{l}\text { Universal Robot Hand } \\
\text { [35] }\end{array}$ & Multicontact Recognition & {$[35]$} \\
\hline Tekscan [57] & Shadow Hand & $\begin{array}{c}\text { Edge Detection, Segmentation, Neural } \\
\text { Networks }\end{array}$ & {$[94]$} \\
\hline $\begin{array}{l}\text { Weiss Robotics sensor } \\
\text { DSA } 9205\end{array}$ & 1 DoF Gripper & Bag-of-Features & [145] \\
\hline Weiss Robotics & $\begin{array}{l}\text { Schunk [109], } \\
\text { ARMAR-IIIb }\end{array}$ & ANN classifier, PCA, SOM & [146] \\
\hline Weiss Robotics & $\begin{array}{l}\text { robotic manipulator } \\
\text { (Phantom Omni) }\end{array}$ & SIFT, $k$-means, $k \mathrm{NN}$, bag-of-features & [147] \\
\hline Tekscan TM(4256 E) & Barret Hand & PCA, convexity, naive Bayes classifier & [148] \\
\hline GelSight [79] & Gripper & $\begin{array}{l}\text { Localization: Binary Robust invariant } \\
\text { scalable keypoints, }\end{array}$ & [149] \\
\hline
\end{tabular}

\subsubsection{Tactile object identification}

Robot fingers with as many sensing modalities as human fingertips, for example the multimodal BioTac sensor [84], can identify an object through its physical properties. In [19], multimodal information is sensed by barometer, thermistor, pressure sensitive liquid, and pattern of electrodes distributed over the entire surface of the fingertip. Artificial neural networks (ANN) and Gaussian mixture model regression (GMMR) are used to extract force vectors from an array of electrodes; those vectors are then used to extract traction information. The barometer gives texture information by analyzing oscillations in the frequency domain. Then the temperature sensor information is combined with these modalities to select exploratory movements to achieve an effective object recognition procedure. Exploratory movements (Table 7) proposed in [43] use Bayesian theory to identify the most informative ac- tion. Although the procedure of object exploration may involve up to six actions, only the first three exploratory movements have been used for object identification. The pressure movement is used to estimate the flexibility of an object. During the lateral sliding motion, a tactile sensor can detect the texture of a surface. Temperature is measured in static contact. The shape of an object can be recognized by calculating the joint angles of the fingers during the enclosure. By lifting an object, the mass of an object can be estimated. Finally, the borders of a surface can be recognized by following the contour. High-passed pressure value of the orthonormal to contact surface electrode is used to explore an object's compliance; around $1.47 \mathrm{~N}$ of force is applied using torque controllers until reaching the steady state. Texture is recognized from vibrations by applying similar computational techniques as in vibration sensing for slip detection (see section 4). Before the slid- 
ing motion, the robot end-effector is controlled by torque controller. When the desired contact force is achieved, the torque controller is switched to mixed position velocity controller to perform the sliding motion. During the sliding motion, the robot gets information about the surface roughness. The traction of a surface can be measured by comparing tangential forces and normal forces. Temperature heat flux is measured by maintaining static contact. After selecting the most informative exploratory movements, objects are classified according to training data.

In other multi-modal approach [139], a 3D visual sensor, auditory information acquired by shaking the object, and tactile images acquired from grasp have been used to identify an object. Statistical model Latent Dirichlet allocation (LDA) is implemented for on-line object categorization.

\subsubsection{Texture Recognition}

Texture recognition, as the vibration-based slip detection techniques 4.1.2, is based on dynamic tactile data and draws on signal processing methods. Most commonly, variations of sensing value, whether they come from microvibration sensors or tactile arrays, are subjected to Fast Fourier transformation (FFT). Then the spectral components and possibly computed features are used for classification algorithms (Figure 20 (a)).

Fishel et al. [22] registered vibrations by change in pressure of a barometer located within a liquid. At initial step, signals from the barometer are filtered by pass-band filter with the bandwidth of from $20 \mathrm{~Hz}$ to $700 \mathrm{~Hz}$. Then Fourier Transformation is applied to the signals. Derived spectral components could be already in the classification algorithm, but would not result in good estimation for properties of a surface. To estimate the roughness of a surface, the authors proposed to calculate a spectral power of the pressure variations, $P_{a c}(n)$ :

$$
\text { Power }=\frac{1}{N} \sum_{n=1}^{N} P_{a c}(n)^{2},
$$

where $\mathrm{N}$ is total number of harmonics. Meanwhile, spectral centroids, SC, used to estimate a fineness of the surface:

$$
S C=\frac{\sum_{n=1}^{N} f f t\left(P_{a c}(n)\right)^{2} * f}{\sum_{n=1}^{N} f f t\left(P_{a c}(n)\right)^{2}},
$$

where $f$ is a frequency, $f f t(\cdot)$ is the FFT. The authors state that these spectral centroids give better estimation of the fineness than conventional relationship $f=v / \lambda$, in which $v$ and $\lambda$ are the velocity and the spatial wavelength of the texture/fingerprints, at a higher velocities or on finer surfaces respectively. These features together with a motor current demand to estimate the tractions of a surface are then implemented in Bayesian classification/exploration (Figure $20(\mathrm{~b})$ ).

In [140] since the authors were limited with the acquisition rate from sensors, the maximum detectable frequency was $100 \mathrm{~Hz}$. The Friction Ratio was estimated by use of a six-axis force and torque sensor. Then variations of a mean squared error (MSE) between the estimated ratio and sensor output were then subjected to FFT. The FFT resulted in spectral components that can be applied in classifications step. The authors applied $k$-NN classifier. (Figure $20(\mathrm{c}))$.

Jamali et al. [142] applied high pass filter with cut-off frequency of $500 \mathrm{~Hz}$ and removed DC (constant) component by use of Zero-Mean Normalization. As in the above approaches the input signal was transformed to frequency domain. The harmonics that occur during sliding were classified by means of Majority voting algorithms (Figure $20(\mathrm{~d}))$.

Unlike the above approaches, Li et al. [79] recognized the texture as an image through a contact pattern sensing the GelSight sensor with a resolution of around $2 \mathrm{mi}-$ crons. The authors proposed Multi-scale local binary pattern (MLBP) to classify high resolution tactile images (Figure $20(\mathrm{e}))$.

Regardless the source of vibrations the applied computational techniques can share common methods. In the approaches proposed by Jamali et al. [142] and Fishel et al. [22], the measured signal, which are acquired from a piezoelectric and liquid pressure sensor, are filtered first and transformed to the frequency domain. Metrics used for the classification are different in these two approaches. While, in the first approach, the authors used the Fourier components as the metrics for a classifier, in the second approach, one more step is taken to extract features that represent surface properties as the metrics for their classifier. The estimation of surface properties from the Fourier components rather than using them directly as the metrics give an advantage to the exploration procedures, because the extracted features can be used to choose the next exploratory action (Table 7 which results in a higher recognition rate.

The vibrations can be also represented by a combination of several variables, as for example, ratio between the normal and tangential forces [140]. The variation of the proposed metric represents the change of the traction properties. Therefore, the used metric are not explicitly related to the surface texture, which may result in not perfect recognition process.

\subsubsection{Contact pattern recognition}

Object recognition from tactile arrays uses image processing techniques [40]. Figure 21 (a) outlines the most common steps in tactile contact pattern recognition: preprocessing, feature extraction, and classification. As preprocessing steps we consider the following operations: spatial filtering, thresholding, and normalization of sensor output values to the highest one. Image features can be computed from tactile images by applying PCA, which results in image moments (i.e. eigenvectors and eigenvalues) that provide information about contact area, center of pressure, and orientation of line in the case of the edge 


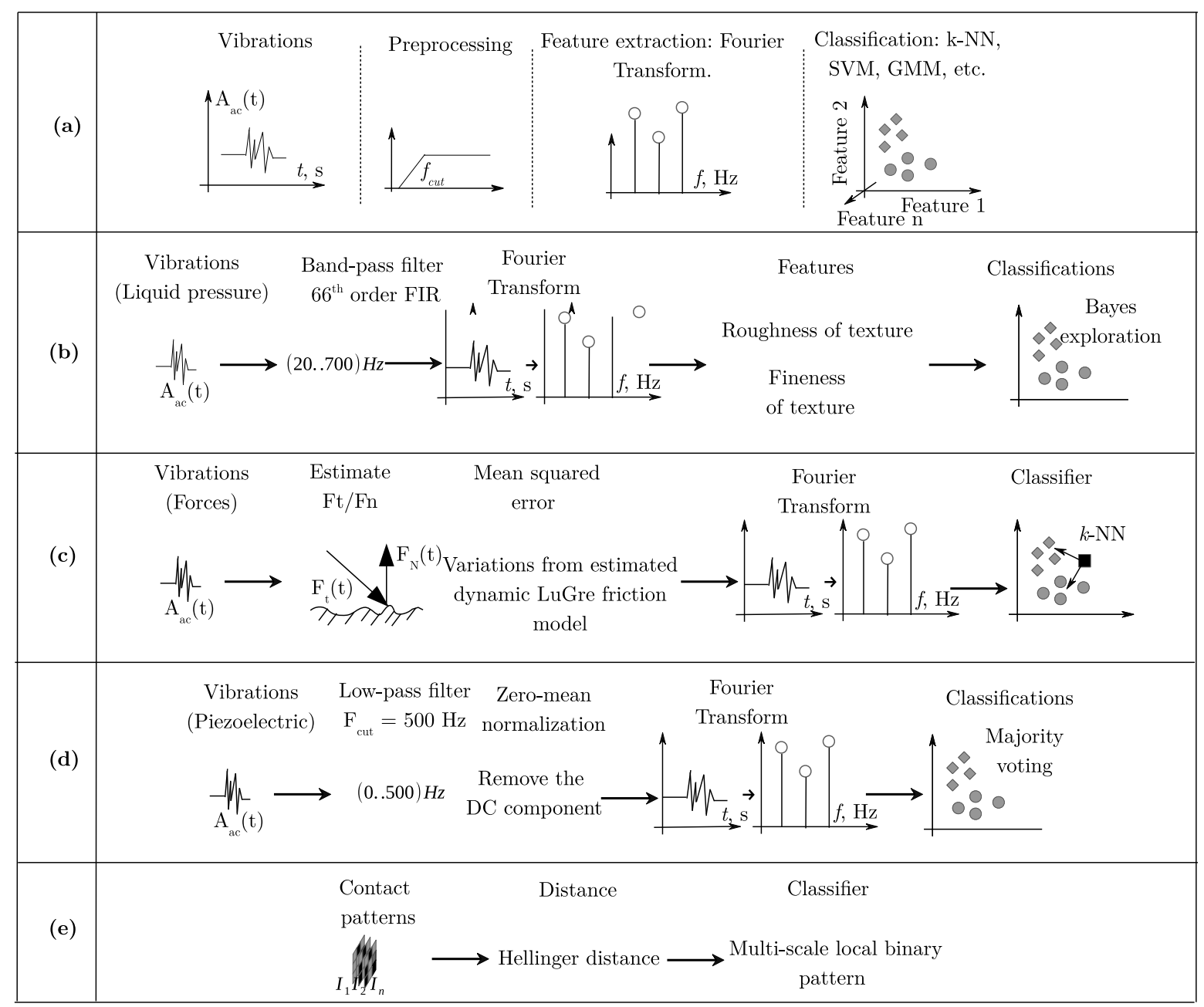

Figure 20: Computational techniques applied in tactile texture recognition: (a) major flow chart of a texture recognition, including filtering, Fourier Transform and feature extraction, and classification; (b) Fishel et al. [22] estimate surface roughness by calculating average spectral power Power from $N$ harmonics with amplitude $P_{a c}(n)$ and surface fineness $\lambda$ by comparing finger velocity $v$ and frequency $f$; (c) Hongbin et al. [140] estimate the dynamic friction model $f_{t} \hat{/} f_{n}$ and detect the variation from the estimated model, where $f_{t}$ and $f_{n}$ are the tangential and normal forces, respectively; (d) Jamali et al. [142] use directly the Fourier components as the feature space for classification algorithm; (e) Li et al. [79] use a Multi-scale local binary pattern, which is operator for texture classification, for contact pattern recognition exploiting the GelSight sensor with a high spatial resolution that allow recognition of even very smooth textures.

contact type. An alternative way of extracting features from a tactile image is to use Hough transformations [152]. This method is less reliable in extracting a straight line as stated in [31] (Figure 22(a)), but can be effectively applied for the detection of circles in the image. Besides geometrical elements, tactile image processing draws on other image processing tools, such as contour detection in order to achieve identification of more complex shapes (Figure 22(b)). Rather than extracting features in spatial units, one could extract image features represented in the frequency domain by applying Fourier Transform. Finally, these features serve as core for classification algorithms. Scale-invariant feature transformation (SIFT), which is mostly used in computer vision, can be also implemented in tactile contact processing to extract features.

Schneider et al. [145] used the bag-of-features approach for tactile pattern recognition. In a preprocessing step, all measurements are normalized to the sensor's maximum response to allow the recognition to be invariant to the pressure level: $Z \in[0 ; 1]^{x * y}$. Two tactile images noted as $I_{1}(x, y)$ and $I_{2}(x, y)$ are compared by computing the Euclidean distance, $d\left(I_{1}, I_{2}\right)$ pixel by pixel:

$$
d\left(I_{1}, I_{2}\right)=\sum_{x} \sum_{y}\left|I_{1}(x, y)-I_{2}(x, y)\right|,
$$

and the distance between two observations, $z 1$ and $z 2$, is calculated by taking into account the distance between fingers, $\omega$, and the weighting factor, $\alpha$, that represents the contribution of changes in contact patterns and finger distance:

$$
\begin{aligned}
d(z 1 ; z 2)= & \alpha *\left(d\left(I_{1}^{\text {left }} ; I_{2}^{\text {left }}\right)+d\left(I_{1}^{\text {right }} ; I_{2}^{\text {right }}\right)\right)+ \\
& +(1-\alpha) *\left|\omega_{1}-\omega_{2}\right|,
\end{aligned}
$$




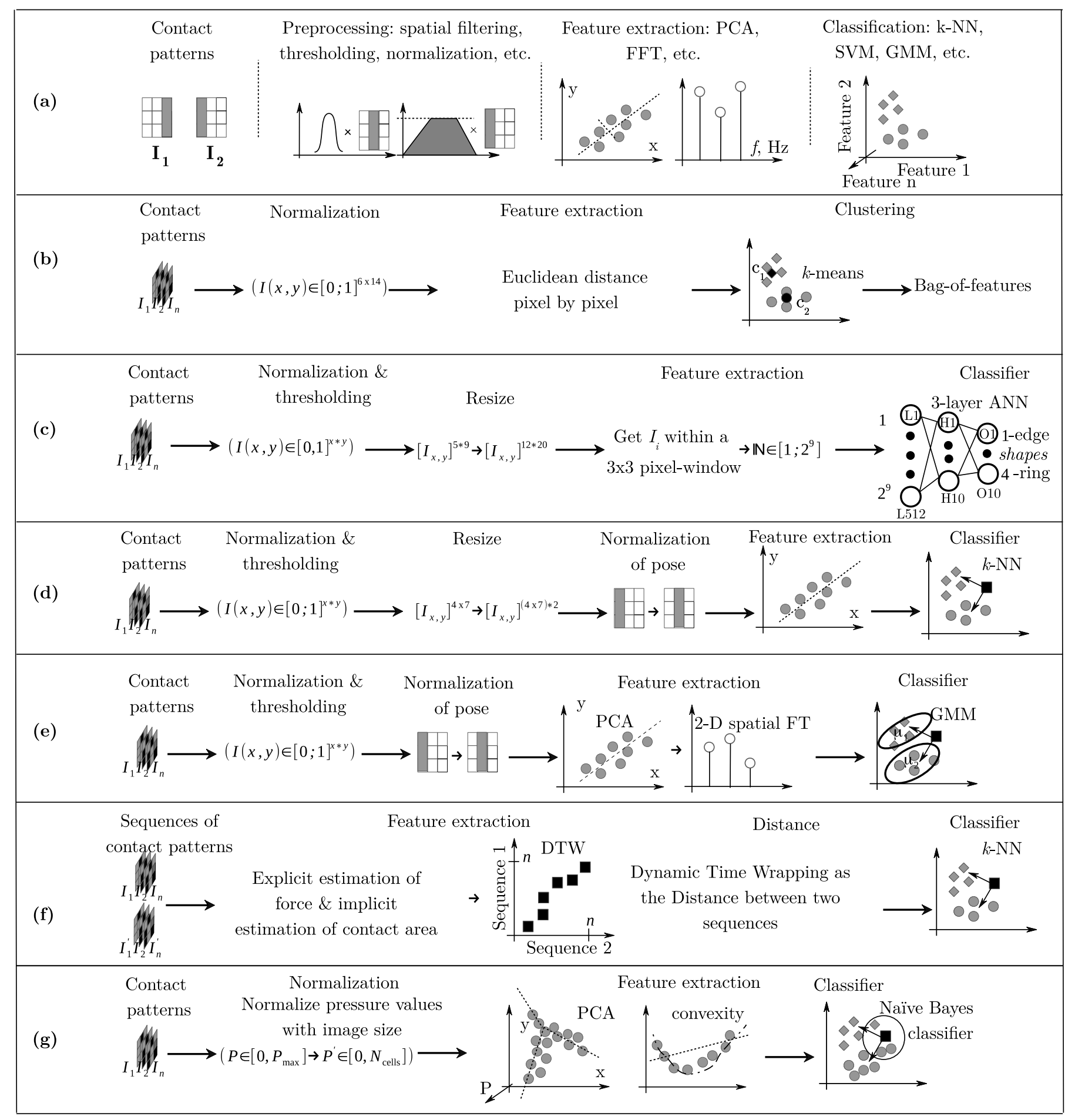

Figure 21: Computational techniques applied in tactile contact pattern recognition: (a) major flow chart of contact pattern recognition, including preprocessing, feature extraction, and classification; (b) Schneider et al. [145] normalize tactile image and calculate Euclidean distance pixel by pixel (c) Hongbin et al. [94], (d) Göger et al. [24], (e) Pezzementi et al. [40], (f) Drimus et al. [49], (g) Hongbin et al. [148]. 
Table 7: Types of actions for object identification [43] based on multimodal tactile perception.

\begin{tabular}{|c|c|c|c|}
\hline $\begin{array}{c}\text { Exploratory } \\
\text { movements }\end{array}$ & $\begin{array}{c}\text { Control } \\
\text { variables }\end{array}$ & $\begin{array}{c}\text { Feedback } \\
\text { signals }\end{array}$ & $\begin{array}{c}\text { Sensory } \\
\text { informa- } \\
\text { tion }\end{array}$ \\
\hline Pressure & $\begin{array}{c}\text { Fingertip } \\
\text { Position }\end{array}$ & $\begin{array}{c}\text { Fingertip } \\
\text { force }\end{array}$ & $\begin{array}{c}\text { Fingertip } \\
\text { deforma- } \\
\text { tion }\end{array}$ \\
\hline $\begin{array}{c}\text { Lateral } \\
\text { sliding }\end{array}$ & $\begin{array}{c}\text { Fingertip } \\
\text { Velocity \& } \\
\text { Force }\end{array}$ & $\begin{array}{c}\text { Fingertip } \\
\text { Velocity \& } \\
\text { Force }\end{array}$ & Vibrations \\
\hline Static & $\begin{array}{c}\text { Fingertip } \\
\text { Pontact }\end{array}$ & $\begin{array}{c}\text { Local de- } \\
\text { formation }\end{array}$ & Heat flow \\
\hline Enclosure & $\begin{array}{c}\text { Hand joint } \\
\text { torques }\end{array}$ & $\begin{array}{c}\text { Hand joint } \\
\text { torques }\end{array}$ & $\begin{array}{c}\text { Hand joint } \\
\text { positions }\end{array}$ \\
\hline Lifting & $\begin{array}{c}\text { Arm joint } \\
\text { position }\end{array}$ & $\begin{array}{c}\text { Arm joint } \\
\text { position }\end{array}$ & $\begin{array}{c}\text { Arm joint } \\
\text { forces }\end{array}$ \\
\hline Contour & Fingertip & Local & Fingertip \\
following & Position & contact & position \\
\hline
\end{tabular}

where $I_{1}^{\text {left }}$ and $I_{1}^{\text {right }}$ stand for tactile images from left and right fingers of a gripper. The $k$-means unsupervised clustering algorithm has been applied to get centers (centroids) of each cluster $\left(c_{1} \ldots c_{k}\right)$. The centroids serve to build a vocabulary for the bag-of-features approach (Figure 21 (b)). To verify the proposed techniques, the authors carried out 830 tactile observations with a 6 x14 piezoresistive array for 21 different objects.

Hongbin et al. [94] applied a three-layer Neural Network to classify contact patterns. As in the above approach, an image is normalized to the highest value in range zero to one. Then two more preprocessing steps of resizing and thresholding operations were carried out. The operation of resizing from a $5 \times 9$ to a $12 \times 20$ image has been implemented by linear interpolation. The thresholding operation provided at the output a binary image. Both operations have been implemented to enhance the tactile image since the sensor used in the paper was with low spatial resolution. In order to get features for the classifier, the authors calculated the number of repetitions of the same image sub-patterns created by sweeping a $3 \times 3$ pixel-window (Figure 21 (c)). The efficacy of the proposed approach has been verified on the recognition of 4 different shapes, including edge, sphere, ring, and rectangle, with 40 tests for each shape.

In addition to thresholding, resizing, and normalization of sensor values during the preprocessing steps, a contact pattern could be also normalized spatially (normalization of a contact pose) as was implemented by Göger et al. [24]. The normalization of a contact pose is performed by means of applying two-dimensional $(p+q)^{t h}$ order image moments, $m_{p, q}$ :

$$
m_{p, q}=\sum_{x} \sum_{y} x^{p} y^{q} I(x, y)
$$

where $\mathrm{x}, \mathrm{y}, \mathrm{I}(\mathrm{x}, \mathrm{y}), \mathrm{p}, \mathrm{q}$ are the two-dimensional coordinates of each tactel in the image, pressure value, $\mathrm{x}$-order, and $\mathrm{y}$ order, respectively. The authors carried out PCA to get a reduced matrix formed by eigenvectors and applied $k$ $\mathrm{NN}$ classifier in a recognition step. For benchmarking, 7 different contacts, including small point, large point, twopoint, full, edge, surface with hole, and waved surface, have been acquired with a $4 \times 7$ array 10 times for training and 10 times for testing. The use of PCA resulted in the matrix containing eigenvectors of the size of 11221 (d).

Pezzementi et al. [40] introduced Moment-Normalized Translation-Invariant descriptor ( feature extractor ), in which the two-dimensional spatial Fourier Transform has been applied to image moments to add invariance to transformations. The authors applied two different clustering algorithms: $k$-means and GMMs; GMMs has shown a higher recognition performance to the detriment of computational time. An image was normalized as well as in approaches described above. An algorithm similar to rapidlyexploring random trees (RRT) has been implemented in the exploratory stage. The recognition and exploration techniques have been tested in simulation of 10 different three-dimensional objects with 100 tactile images per object. As in the above preprocessing steps, the images are first normalized, resized by factor 2 to enhance the quality of the image due to the low resolution of $4 \times 7$, and thresholded for calculation of image moments 21 (e).

As tactile contact patterns change with the time when a robot squeezes a deformable object, one could extract a set of features from a series of images from one tactile array. Drimus et al. [49] proposed to use an explicit estimate of an average pressure:

$$
P_{a v g}=\frac{1}{N_{x} * N_{y}} \sum_{x} \sum_{y} I(x, y),
$$

in which $N_{x}$ and $N_{y}$ are the number of sensing cells in row and column of an array, and an implicit estimate of contact area:

$$
\text { area }=\sqrt{\frac{1}{N_{x} * N_{y}} \sum_{x} \sum_{y}\left(I(x, y)-P_{a v g}\right)^{2}}
$$

as the features to recognize deformable objects. Then Dynamic Time Wrapping (DTW) applied to these features in order to find the shortest path between two tactile images from the same tactile sensor in two consequent moments of time. As in the approach proposed by Schneider et al. [145] and described above, the authors calculate the Euclidean distance between two observations $z_{1}, z_{2}$. However, the gripping distance is not taken into account and the distance between two observations is calculated by means of 
DTW, applied on the features, when in the former approach the distance is directly calculated in image space:

$$
d\left(z_{1}, z_{2}\right)=D T W\left(P_{\text {avg }}^{1}, P_{\text {avg }}^{2}\right) .
$$

Similar to Göger et al. [24], the $k$-NN classifier has been carried out in a recognition step. It was shown that a robot exploiting the above algorithm could distinguish a spoilt fruit from a fresh fruit by applying palpations with a two-fingered gripper and an $8 \times 8$ sensing array (Figure 21 (f).

In [148], instead of converting a tactile image to a binary image, authors apply PCA to a pressure profile and extract orthonormal eigenvectors in the three-dimensional space. In addition to these vectors (3 principal axis of a profile), convexity and concavity of the pressure profile are estimated by comparing a pressure value at a centroid (center of pressure) and its surrounding area. Then contact patterns are classified by applying naive Bayes approach to this set of features. Pressure values are normalized and scaled to the range that is equivalent to that of the number of sensing cells in $\mathrm{x}$ and $\mathrm{y}$. Benchmarking has been performed on recognition of 6 contact patterns with a 5x9 sensing array (Figure $21(\mathrm{~g})$.

A rather high precision, $0.14 \mathrm{~mm}$, in localization of an object in a hand has been achieved by use of GelSight sensor [149] and by means of Binary Robust invariant scalable keypoints (BRISK).

Several approaches use the same preprocessing and feature extraction methods as it can be noticed from Figure 21. When the spatial resolution of sensing arrays is not high enough, the contact image is resized and augmented by various interpolation methods (Figure 21 (c, d)). Then the features can be extracted with a higher accuracy.

In the most of the cases, only the shape of the contact pattern is of importance and, therefore, pressure values can be normalized. However, it is not applicable in recognition of deformable objects (Figure 21 (f)), because pressured values used to estimate an average force.

Thresholding operation that result in a binary image improves performance of feature extractors and used in the approaches, in which two-dimensional PCA is applied to get metrics for classifiers (Figure $21(\mathrm{~d}, \mathrm{e})$ ). In the cases when the Euclidean distance between tactels is used as the metric (Figure 21 (b)) or the three-dimensional contact profile is of importance (Figure $21(\mathrm{~g})$ ), the thresholding operation can not be used.

\subsection{Tactile Servoing}

Humans often perform tactile servoing actions almost subconsciously, for example, when they search in a pocket a key. In cases when visual information is not available, the motor responses are coupled with tactile feedback only. In autonomous robot control theory, tactile feedback can be used to servo objects. The concept of tactile servoing is analogous to image-based visual servoing [153], [154]. Robot motion driven by tactile feedback was implemented

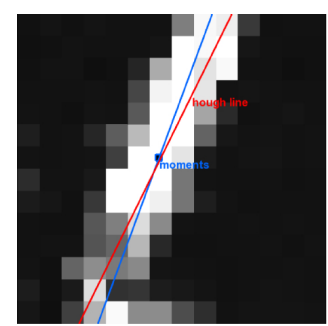

(a)

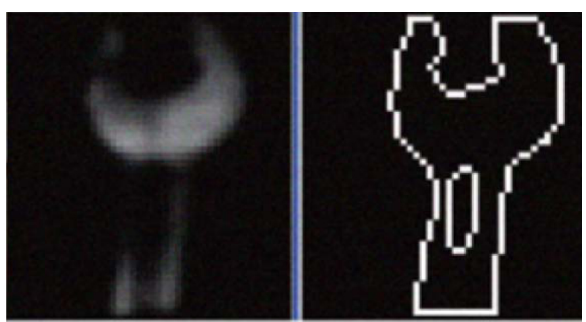

(b)
Figure 22: Contact pattern recognition and feature extraction from a tactile image: (a) extracted feature of contact edge based on image moments (blue line) and hough line transform (red line) [31]; (b) geometrical shape derived from the tactile image: original tactile image on the left and detected contour on the right [133].

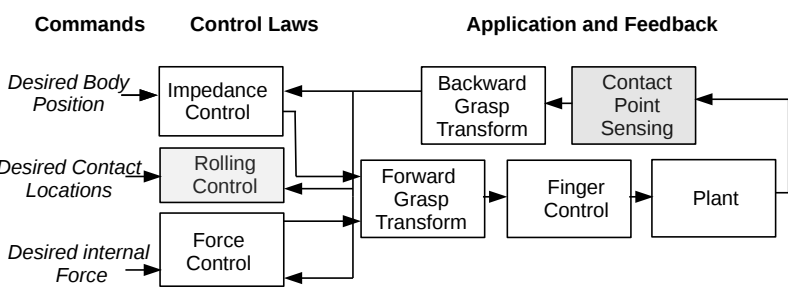

Figure 23: General control framework for dexterous manipulation [157]. The path of information from command through control laws to application on the dexterous hand using object impedance control for rolling manipulation is shown. The highlighted blocks can be replaced with tactile servoing control laws.

by Berger et al. [155] and then extended to tactile servo concept by Sikka et al. [156] in the early 1990s. However, tactile servoing is not well investigated, which is partially due to the fact that tactile perception technology is not as well developed as vision technology. A good resolution and a high number of tactels in tactile array are needed to improve the performance of tactile servoing algorithms.

In dexterous manipulation tasks, tactile servoing can be assigned to the middle level of the control architecture as proposed by Okamura et al. [157] for dexterous end-effectors. The authors determined three levels of control for dexterous manipulation: low-Level is for impedance and active compliance control, kinematics, and forces; mid-Level is responsible for manipulation phases, transitions between the manipulation phases, and event (i.e. contact) detection; high-Level is dedicated to planning a task and choosing a grasp (Figure 23).

In the current state-of-the-art, tactile servo schemes have been proposed for only planar end-effectors with single tactile array [31], [158]. Objects in the real world can have many different shapes, but the types of contact that have been detected so far are limited to a few particular cases: plane on plane, line on plane, and point on plane.

An example of a basic tactile servoing control architecture is shown in Figure 24. The motion planner specifies the desired contact state, $S_{d}$. Extracted features from the 


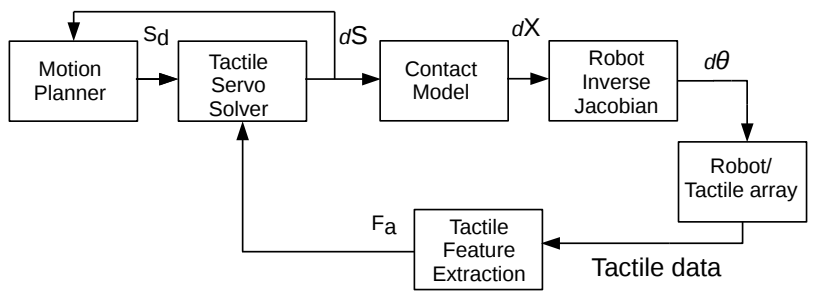

Figure 24: Architecture of a tactile servoing controller [158]. The tactile feature extraction processes the contact information and provides features to control. Desired contact state, $S_{d}$, is compared with the actual contact state, $S_{a} . S_{a}$ is derived from actual tactile feature, $F_{a}$. The errors in the contact state space are transformed to the joint space of a robot.

tactile sensor, $F_{a}$, are transformed to actual contact state, $S_{a}$, when inverse sensor model is available; then $S_{a}$ is compared with $S_{d}$ to generate the error. If an inverse sensor model is not available, then the Tactile Jacobian is needed to relate the variation in the tactile feature vector to that in the contact state. The tactile servo solver generates an error, $d S$, and informs the planner about the adjustment of the desired contact state. The contact model block is dedicated to transform the changes from the contact feature state to the position of the robot's end-effector $d X$ in the task space. Finally, the Robot Inverse Jacobian is applied to calculate the robot's joint values $d \theta$ from the error of the end-effector position in Cartesian space. The robot joint angles calculated via tactile feedback are expressed as follows [159]:

$$
\begin{aligned}
& \theta(t+1)=\theta(t)+d \theta, \\
& d \theta=J_{\theta}^{-1} d X, \\
& d X=\frac{X_{f}-X_{a}(t)}{T_{\text {seg }}-t}\left(t<T_{\text {seg }}\right), \\
& X_{a}(t)=f_{s}^{-1}\left(F_{a}(t)\right),
\end{aligned}
$$

where $\theta(t)$ and $\theta(t+1)$ are the actual and calculated joint angles, $d \theta$ is the error in joint angles, $f_{s}^{-1}(\cdot)$ is the inverse tactile model, $J_{\theta}^{-1}(\cdot)$ is the robot inverse kinematics, $X_{a}$ is the actual Cartesian position of the robot end-effector, $X_{f}$ is the desired final position, $T_{\text {seg }}$ and $t$ are the period of time within which the robot reaches its final position and time.

In [31], this tactile servo controller is extended by implementing a selection matrix for combining various servoing tasks, i.e. following an edge with a tactile array attached to the planar end-effector or aligning the orientation with a detected edge. The inverted task Jacobian was introduced to transform errors in the space of tactile image features into errors in a motion twist in the Cartesian space. Unlike the feature extraction through inverse tactile sensor model in the former control scheme, tactile features in the latter one are extracted using image processing techniques such as PCA and Hough transformations. The extracted tactile image features are then transformed to

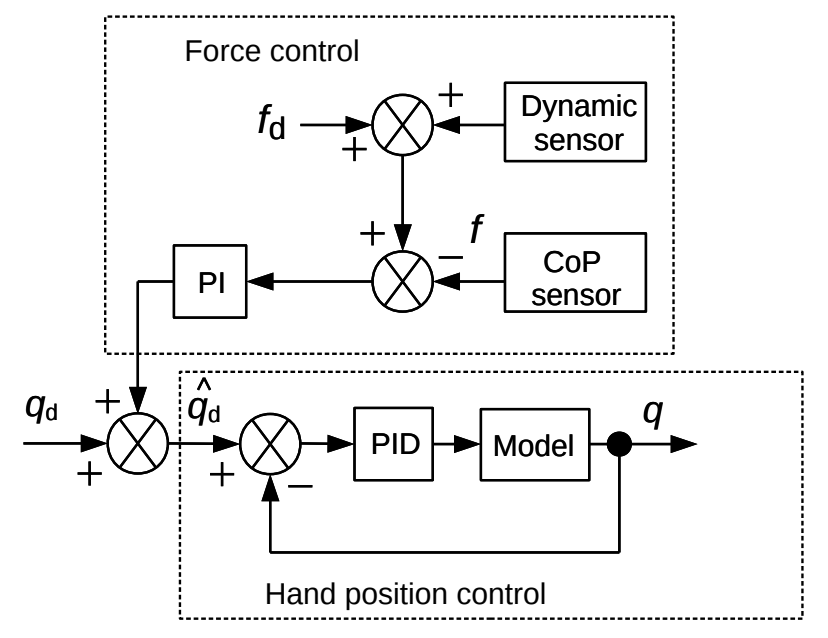

Figure 25: Block diagram of position-based force control using tactile sensors [39]. The position of a hand is modified according to the measured force and detected slip signal. The slip signal increases the desired force. $q_{d}, \hat{q_{d}}$, and $q$ are the desired joint angle, modified desired joint angle, and actual joint angle, respectively. $f_{d}$ and $f$ are the desired and actual forces. The desired force increases when the dynamic sensor detects vibrations.

motions:

- positional deviations are mapped into tangential motions in $\mathrm{x}$ - and $\mathrm{y}$ - directions w.r.t the sensor frame;

- normal forces are mapped to motion in z-orientation w.r.t the sensor frame;

- rotational error is mapped into rotational velocity around z-axis w.r.t the sensor frame.

In [23], tactile information from a fingertip of the iCUB humanoid robot is used to follow an edge of an object by performing palpations. It was shown that the robot could follow sophisticated contours when the recognition of the contact pattern was performed after each palpation.

As the touch driven control algorithms are important in exploratory actions, tactile servoing plays an essential role the exploration of unknown objects. However, due to imperfections of tactile sensing arrays, difficulties in integration of these arrays, and computational costs, which impede to process the data to use within the control loops. Tactile servoing [155] has been implemented on planar endeffectors only [31].

\subsection{Force Control with Tactile Sensors}

In the robotics literature, tactile feedback, being mainly used for event-driven manipulation [124], has been rarely employed inside a control loop because of the noisy signals coming from tactile sensors [8]. Most of the research in force control with dexterous hands is aimed at controlling the grasping force in order to achieve a stable grasp (Section 4.1). Li et al. [31] control the normal force to keep the robot in contact with an object during the edge servoing task. Force and a Center of Pressure of the contact 


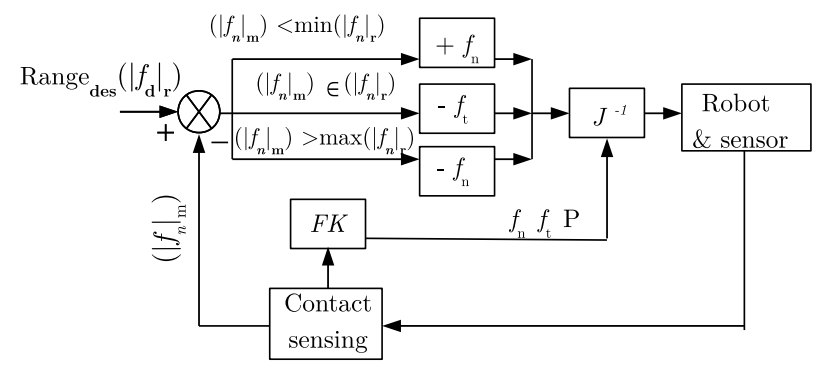

Figure 26: Surface contour following using the contact sensing finger (control diagram). [160]. Range des $\left(\left|f_{d}\right|_{r}\right)$ is a range of the desired force, $\left(\left|f_{n}\right|_{m}\right)$ is the measured normal force, $\left(\left|f_{n}\right|_{r}\right)$ is the friction cone, $\left(f_{n}\right)$ and $\left(f_{t}\right)$ are the normal and tangential forces, $\mathrm{P}$ is the contact point location, and $\left(J^{-1}\right)$ is the robot's inverse kinematics.

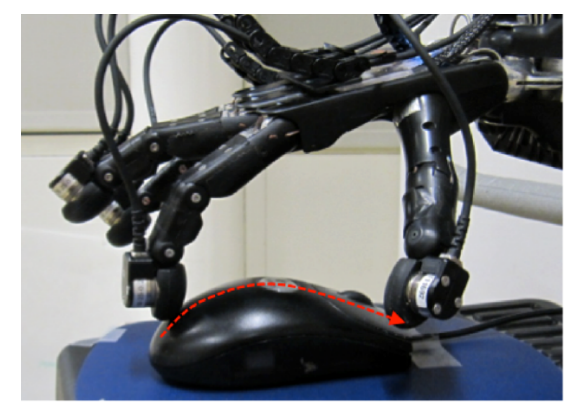

Figure 27: Hand with ellipsoid f/t sensors following a surface (the red dashed line) [104].

pattern are estimated by following equations:

$$
\begin{aligned}
& c=f^{-1} \sum_{i, j \in R} f_{i j} c_{i j} ; \\
& f=\sum_{i, j \in R} f_{i j},
\end{aligned}
$$

where $c_{i j}$ are the discrete coordinates of the tactels, $f$ and $f_{i j}$ are the total force and force of each tactel, respectively, and $R$ is the number of tactels in $\mathrm{x}$ - an $\mathrm{y}$-directions.

Theshigivara et al. [39] obtain signals from both a dynamic tactile array and a Center of Pressure (CoP) tactile sensor to control the grasping force (Figure 25). The desired force $f_{d}$ increases proportionally to the spectral power of the vibrations that occur during the slip. Vibrations are measured by dynamic tactile sensors. The actual force, which is measured by CoP, is then subtracted the modified desired force. The desired joint angle $q_{d}$ is then calculated according to the force and vibrations.

In [38], tactile information derived from one type of tactile sensors only is used to control both grasping force and slippage. The contact force is calculated according to equation 10. During grasping, the desired force is compared to actual forces of tactile sensors. In the lift and hold phase, the applied force increases proportionally to the actual force at each instance of the slip signal.

The above force controllers are used to ensure stable grasping. Another application of tactile sensors in force control include the surface following motion. Following unknown surface is an essential task to explore objects. An advanced fingertip sensor with capability to measure contact point locations, e.g. [104], could be used to accomplish such type of tasks. When the contact surface of a fingertip is known, the contact location with the environment can be estimated by measuring forces and torques in $\mathrm{SE}(3)$ [160]. A desired friction cone is given, and if the normal force $\mathrm{Fn}$ is smaller than the smallest value of a range, a finger moves towards the contact, and vice versa. If the $F_{n}$ is in the range, then the finger moves in the sliding direction only. Errors in contact locations and forces are transformed to joint angles $q$ through Jacobian matrix $J$ (Figure 26). A result of exploiting the controller for following a surface with a fingertip of a robot hand is shown in Figure 27(red dashed line).

\section{Conclusions and Future Research}

Applications of tactile sensing in autonomous manipulation and research trends in this field over the last two decades have been reviewed. The major tactile sensor types and computational techniques have been discussed. Table 8 summarizes and compare the usefulnesses of different tactile data types in the reviewed robot hand applications.

Despite all the advances in sensor technologies and their integration in robotic hands (described in Sections 2 and 3.1) and the development of new techniques to process and interpret the data provided by them (described in Section 4), there is still a wide scope of investigation in the field of autonomous dexterous manipulation based on tactile sensing. Research to be undertaken in the future includes the following:

1) Design of dexterous robot hands with integrated tactile sensors. Wires can be routed within the structure of the robot hands to ensure efficient connection of embedded sensors. The non-linear frictions in the tendon driven robot hands and backlash of the actuators should be reduced to enhance manipulation performance. In the case of electric robotic hands, tactile sensors are usually affected by the noise of the electric motors actuating the fingers [22]. In addition, mechanical vibrations transmitted by the motors can interfere with vibration-based sensors [38] for slippage detection. Therefore, new materials (such as the fluidic hand [24]) and new isolation solutions should be considered in order to remove these problems.

2) Multimodal object recognition exploiting geometric model of the robot hands. Presence of multimodal information requires algorithms that combine different perception modalities. Fusion of visual and tactile data can be used for more precise estimation of the robot's pose in the world [161]. By considering tactile sensing arrays and the structure of the hands, the shape recognition process can be significantly accelerated compared to the single contact tactile shape recognition when only one tactile sensor 


\begin{tabular}{|c|c|c|c|c|}
\hline 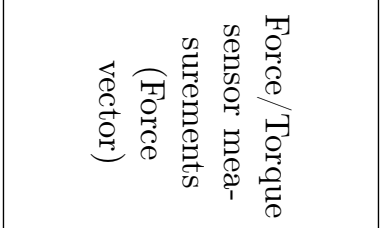 & 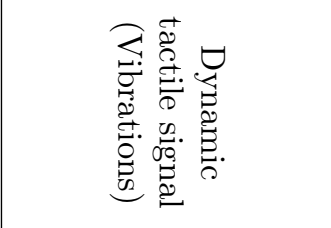 & 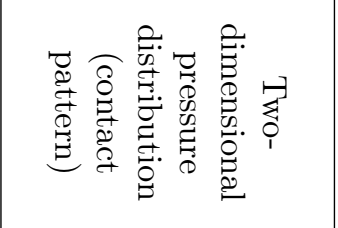 & & \\
\hline 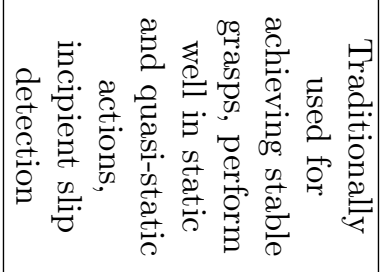 & 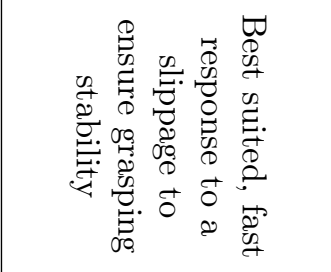 & 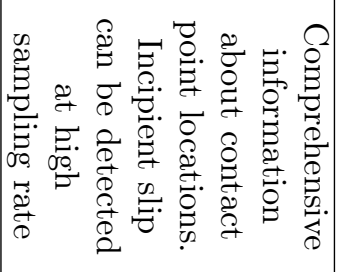 & & \\
\hline 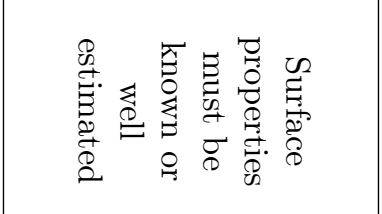 & 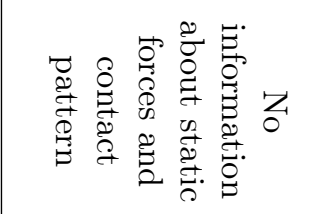 & 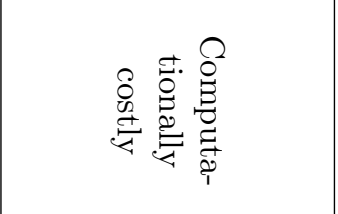 & & \\
\hline 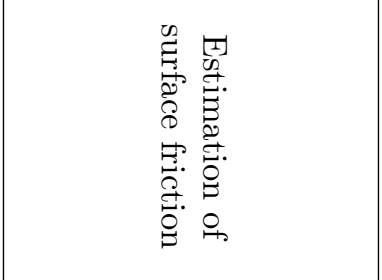 & 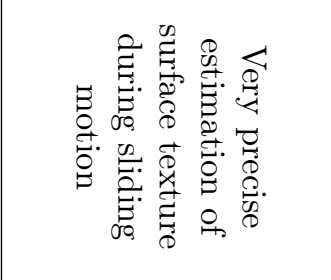 & 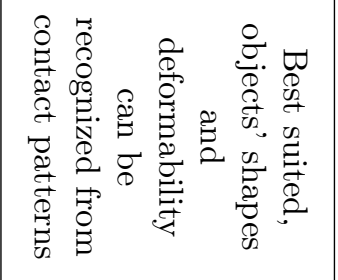 & & \\
\hline 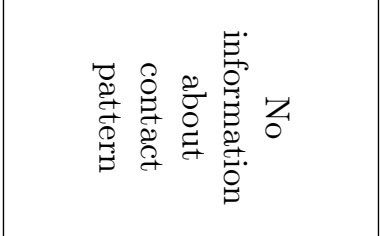 & 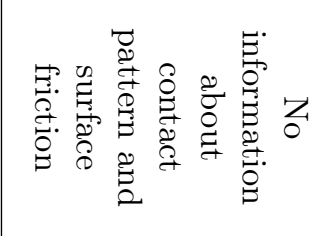 & 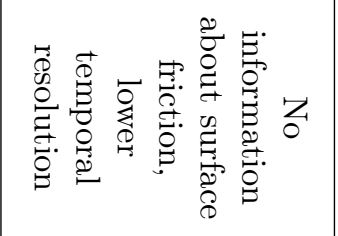 & & \\
\hline 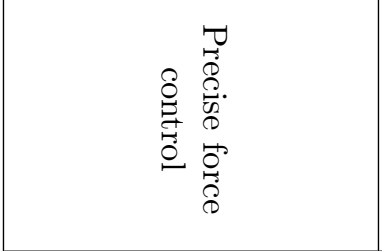 & $\begin{array}{l}2 \\
\text { L }\end{array}$ & 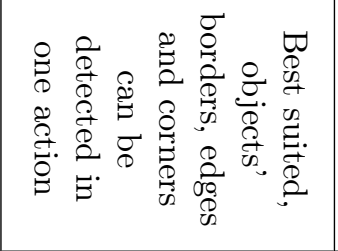 & & \\
\hline 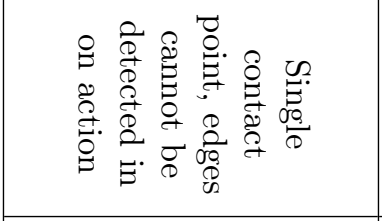 & $\begin{array}{l}2 \\
\text { Z }\end{array}$ & 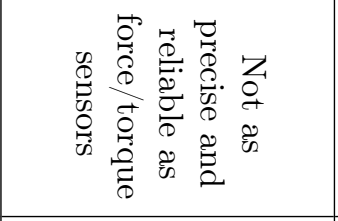 & & \\
\hline 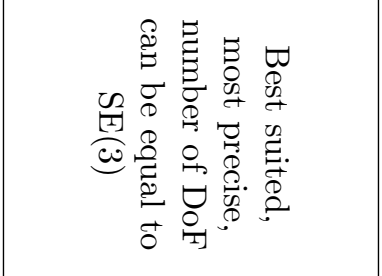 & 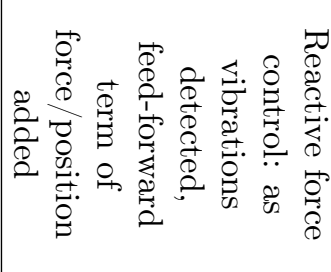 & 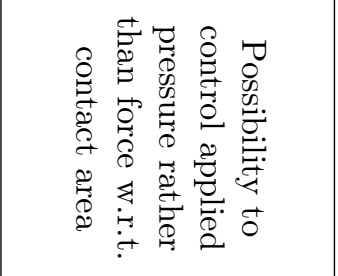 & & \\
\hline 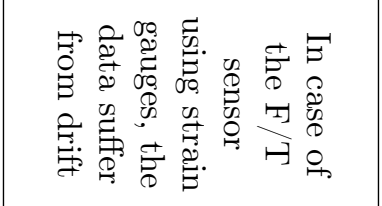 & 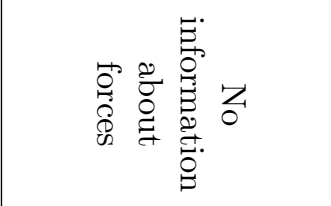 & 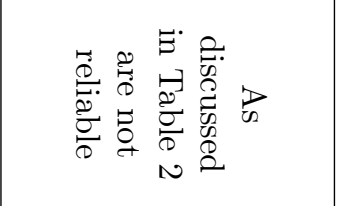 & & \\
\hline
\end{tabular}


explores an object surface. However, most of the tactile object recognition approaches do not take into account complex hand geometry so far.

Moreover, finding common features between visual and tactile sensing modalities is a key point for smooth transition from visual-based to tactile-based control during the exploration of unknown objects.

3) Reactive behavior with dynamic data. Although force control algorithms (for example, hybrid position/force control by Raibert and Craig [162]) have been investigated since the early 1990s, there are a lot of unsolved problems in grasping and autonomous manipulation tasks. In experimental results from the DARPA Autonomous Robotic Manipulation program, Righetti et al. [2] have shown that the robot could not react fast enough to unexpected disturbances, an issue related to reactive behaviors. This problem can be solved by dynamic tactile sensing, as it was reviewed in section 4.1.2. Grasping based on the analysis of vibrations can be applied to address the problem of reactive behavior.

4) Measurement of tangential forces. Slip detection techniques based on friction force models do not suffer from frequency interference as do vibration-based techniques. However, friction force models require measurements of both the normal and tangential reaction forces, which cannot be retrieved from most of the pressure sensing tactile sensors.

5) In-hand manipulation algorithms for both rigid and deformable objects. In-hand manipulation has become increasingly important in recent years. This task requires a lot of computational effort, since the number of DoF of the dexterous robot hand is as much as the whole body of a humanoid robot. In-hand object manipulation is not sufficiently addressed by current research and is one of the areas that warrants further investigation.

Future research work in dexterous manipulation should be focused on the investigation of autonomous control algorithms that comprehensively use tactile feedback by applying tactile servoing and force controls, as well as on multimodal object recognition and tactile-based stable grasp estimation in order to enhance the performance of dexterous manipulation and allow robots to operate in real world with a highly modifying environment.

[1] A. Bicchi, Hands for dexterous manipulation and robust grasping: a difficult road toward simplicity, Robotics and Automation, IEEE Transactions on 16 (6) (2000) 652-662. doi: 10.1109/70.897777.

[2] L. Righetti, M. Kalakrishnan, P. Pastor, J. Binney, J. Kelly, R. Voorhies, G. Sukhatme, S. Schaal, An autonomous manipulation system based on force control and optimization, Autonomous Robots 36 (1-2) (2014) 11-30. doi:10.1007/ s10514-013-9365-9.

[3] B. Siciliano, O. Khatib (Eds.), Springer Handbook of Robotics, Springer, 2008. doi:10.1007/978-3-540-30301-5.

[4] euRobotics aisbl, Robotics 2020. strategic research agenda for robotics in europe, http://www.eu-robotics.net/cms/ upload/PDF/SRA2020_0v42b_Printable_.pdf, accessed May $26,2014$.

[5] B. Gates, A robot in every home, Scientific American 296 (1) (2007) 58-65.
[6] R. S. Johansson, J. R. Flanagan, Coding and use of tactile signals from the fingertips in object manipulation tasks, Nature Reviews Neuroscience 10 (5) (2009) 345-359. doi:10.1038/ nrn2621.

[7] J. R. Wingert, H. Burton, R. J. Sinclair, J. E. Brunstrom, D. L. Damiano, Tactile sensory abilities in cerebral palsy: deficits in roughness and object discrimination, Developmental Medicine \& Child Neurology 50 (11) (2008) 832-838. doi:10.1111/j. 1469-8749.2008.03105.x.

[8] M. Prats, A. P. del Pobil, P. J. Sanz, Robot physical interaction through the combination of vision, tactile and force feedback, in: B. Siciliano, O. Khatib (Eds.), Tracts in Advanced Robotics, Vol. 84 of Springer Tracts in Advanced Robotics, Springer, 2013, p. 177. doi:10.1007/978-3-319-03017-3_3.

[9] M. R. Cutkosky, J. Ulmen, Dynamic tactile sensing, in: R. Balasubramanian, V. J. Santos (Eds.), The Human Hand as an Inspiration for Robot Hand Development, Vol. 95 of Springer Tracts in Advanced Robotics, Springer-Verlag, 2014, pp. 219 246. doi:10.1007/978-3-319-03017-3_3.

[10] R. Dahiya, M. Valle, Tactile sensing technologies, in: Robotic Tactile Sensing, Springer Netherlands, 2013, pp. 79-136. doi: 10.1007/978-94-007-0579-1_5.

[11] T. Sekitani, U. Zschieschang, H. Klauk, T. Someya, Flexible organic transistors and circuits with extreme bending stability, Nature materials 9 (12) (2010) 1015-1022.

[12] M. Kaltenbrunner, T. Sekitani, J. Reeder, T. Yokota, K. Kuribara, T. Tokuhara, M. Drack, R. Schwödiauer, I. Graz, S. Bauer-Gogonea, et al., An ultra-lightweight design for imperceptible plastic electronics, Nature 499 (7459) (2013) 458 463.

[13] H. Yousef, M. Boukallel, K. Althoefer, Tactile sensing for dexterous in-hand manipulation in robotics - a review, Sensors and Actuators A: Physical 167 (2) (2011) 171 - 187, solid-State Sensors, Actuators and Microsystems Workshop. doi:http://dx.doi.org/10.1016/j.sna.2011.02.038.

[14] P. Puangmali, K. Althoefer, L. Seneviratne, D. Murphy, P. Dasgupta, State-of-the-art in force and tactile sensing for minimally invasive surgery, Sensors Journal, IEEE 8 (4) (2008) 371-381. doi:10.1109/JSEN. 2008.917481.

[15] M. I. Tiwana, S. J. Redmond, N. H. Lovell, A review of tactile sensing technologies with applications in biomedical engineering, Sensors and Actuators A: Physical 179 (0) (2012) 17 31.

[16] M. Francomano, D. Accoto, E. Guglielmelli, Artificial sense of slip - a review, Sensors Journal, IEEE 13 (7) (2013) 2489-2498. doi : 10.1109/JSEN. 2013.2252890.

[17] R. Dahiya, G. Metta, M. Valle, G. Sandini, Tactile sensing from humans to humanoids, Robotics, IEEE Transactions on 26 (1) (2010) 1-20. doi:10.1109/TRO.2009.2033627.

[18] R. Dahiya, P. Mittendorfer, M. Valle, G. Cheng, V. Lumelsky, Directions toward effective utilization of tactile skin: A review, Sensors Journal, IEEE 13 (11) (2013) 4121-4138. doi:10. 1109/JSEN . 2013. 2279056.

[19] N. Wettels, J. Fishel, G. Loeb, Multimodal tactile sensor, in: R. Balasubramanian, V. J. Santos (Eds.), The Human Hand as an Inspiration for Robot Hand Development, Vol. 95 of Springer Tracts in Advanced Robotics, Springer International Publishing, 2014, pp. 405-429. doi:10.1007/ 978-3-319-03017-3_19.

[20] M. Lee, H. Nicholls, Review article tactile sensing for mechatronics - a state of the art survey, Mechatronics 9 (1) (1999) 1 - 31. doi:http://dx.doi.org/10.1016/S0957-4158(98) $00045-2$.

[21] S. Navarro, M. Marufo, Y. Ding, S. Puls, D. Goger, B. Hein, H. Worn, Methods for safe human-robot-interaction using capacitive tactile proximity sensors, in: Intelligent Robots and Systems (IROS), 2013 IEEE/RSJ International Conference on, 2013, pp. 1149-1154. doi:10.1109/IROS.2013.6696495.

[22] J. A. Fishel, G. E. Loeb, Bayesian exploration for intelligent identification of textures, Frontiers in Neurorobotics 6 (4). doi : 10.3389/fnbot . 2012.00004 . 
[23] U. Martinez-Hernandez, T. Dodd, L. Natale, G. Metta, T. Prescott, N. Lepora, Active contour following to explore object shape with robot touch, in: World Haptics Conference (WHC), 2013, 2013, pp. 341-346. doi:10.1109/WHC.2013. 6548432 .

[24] D. Göger, N. Gorges, H. Worn, Tactile sensing for an anthropomorphic robotic hand: Hardware and signal processing, in: Robotics and Automation, 2009. ICRA '09. IEEE International Conference on, 2009, pp. 895-901. doi:10.1109/ROBOT. 2009. 5152650.

[25] H. Dang, P. Allen, Stable grasping under pose uncertainty using tactile feedback, Autonomous Robots 36 (4) (2014) 309330. doi: $10.1007 / \mathrm{s} 10514-013-9355-\mathrm{y}$.

[26] H. Liu, X. Song, J. Bimbo, K. Althoefer, L. Senerivatne, Intelligent fingertip sensing for contact information identification, in: J. S. Dai, M. Zoppi, X. Kong (Eds.), Advances in Reconfigurable Mechanisms and Robots I, Springer London, 2012, pp. 599-608. doi :10.1007/978-1-4471-4141-9_54.

[27] X. Song, H. Liu, K. Althoefer, T. Nanayakkara, L. Seneviratne, Efficient break-away friction ratio and slip prediction based on haptic surface exploration, Robotics, IEEE Transactions on 30 (1) (2014) 203-219. doi:10.1109/TRO. 2013.2279630.

[28] K.-C. Nguyen, V. Perdereau, Fingertip force control based on max torque adjustment for dexterous manipulation of an anthropomorphic hand, in: Intelligent Robots and Systems (IROS), 2013 IEEE/RSJ International Conference on, 2013, pp. 3557-3563. doi:10.1109/IROS.2013.6696863.

[29] L. D. Harmon, Automated tactile sensing, The International Journal of Robotics Research 1 (2) (1982) 3-32. doi:10.1177/ 027836498200100201.

[30] J. A. C. Ramon, V. Perdereau, F. T. Medina, Multi-fingered robotic hand planner for object reconfiguration through a rolling contact evolution model, in: 2013 IEEE International Conference on Robotics and Automation, Karlsruhe, Germany, May 6-10, 2013, 2013, pp. 625-630. doi:10.1109/ICRA.2013. 6630638.

[31] Q. Li, C. Schürmann, R. Haschke, H. Ritter, A control framework for tactile servoing, in: Robotics: Science and Systems, 2013.

[32] P. J. Kyberd, M. Evans, S. te Winkel, An intelligent anthropomorphic hand, with automatic grasp, Robotica 16 (1998) $531-536$.

[33] M. Cutkosky, R. Howe, W. Provancher, Force and tactile sensors, in: B. Siciliano, O. Khatib (Eds.), Springer Handbook of Robotics, Springer Berlin Heidelberg, 2008, pp. 455-476. doi: 10.1007/978-3-540-30301-5_20.

[34] R. Koiva, M. Zenker, C. Schurmann, R. Haschke, H. Ritter, A highly sensitive $3 \mathrm{~d}$-shaped tactile sensor, in: Advanced Intelligent Mechatronics (AIM), 2013 IEEE/ASME International Conference on, 2013, pp. 1084-1089. doi:10.1109/AIM. 2013. 6584238.

[35] W. Fukui, F. Kobayashi, F. Kojima, H. Nakamoto, N. Imamura, T. Maeda, H. Shirasawa, High-speed tactile sensing for array-type tactile sensor and object manipulation based on tactile information, Journal of Robotics 2011.

[36] D. Gunji, Y. Mizoguchi, S. Teshigawara, A. Ming, A. Namiki, M. Ishikawaand, M. Shimojo, Grasping force control of multifingered robot hand based on slip detection using tactile sensor, in: Robotics and Automation, 2008. ICRA 2008. IEEE International Conference on, 2008, pp. 2605-2610. doi:10.1109/ ROBOT . 2008.4543605.

[37] H. Yussof, M. Ohka, H. Suzuki, N. Morisawa, Tactile sensingbased control system for dexterous robot manipulation, in: S.I. Ao, B. Rieger, S.-S. Chen (Eds.), Advances in Computational Algorithms and Data Analysis, Vol. 14 of Lecture Notes in Electrical Engineering, Springer Netherlands, 2009, pp. 199213. doi:10.1007/978-1-4020-8919-0_15.

[38] J. Romano, K. Hsiao, G. Niemeyer, S. Chitta, K. Kuchenbecker, Human-inspired robotic grasp control with tactile sensing, Robotics, IEEE Transactions on 27 (6) (2011) 1067-1079. doi : 10.1109/TRO.2011.2162271.
[39] S. Teshigawara, T. Tsutsumi, S. Shimizu, Y. Suzuki, A. Ming, M. Ishikawa, M. Shimojo, Highly sensitive sensor for detection of initial slip and its application in a multi-fingered robot hand, in: Robotics and Automation (ICRA), 2011 IEEE International Conference on, 2011, pp. 1097-1102. doi:10.1109/ ICRA. 2011.5979750.

[40] Z. Pezzementi, E. Plaku, C. Reyda, G. Hager, Tactile-object recognition from appearance information, Robotics, IEEE Transactions on 27 (3) (2011) 473-487. doi:10.1109/TRO. 2011.2125350.

[41] P. J. Kyberd, P. H. Chappell, Object-slip detection during manipulation using a derived force vector, Mechatronics 2 (1) (1992) $1-13$.

[42] L. U. Odhner, L. P. Jentoft, M. R. Claffee, N. Corson, Y. Tenzer, R. R. Ma, M. Buehler, R. Kohout, R. D. Howe, A. M. Dollar, A compliant, underactuated hand for robust manipulation, The International Journal of Robotics Researchdoi: 10.1177/0278364913514466.

[43] D. Xu, G. Loeb, J. Fishel, Tactile identification of objects using bayesian exploration, in: Robotics and Automation (ICRA), 2013 IEEE International Conference on, 2013, pp. 3056-3061. doi : 10.1109/ICRA. 2013.6631001.

[44] A. Schmitz, P. Maiolino, M. Maggiali, L. Natale, G. Cannata, G. Metta, Methods and technologies for the implementation of large-scale robot tactile sensors, Robotics, IEEE Transactions on 27 (3) (2011) 389-400. doi:10.1109/TRO.2011.2132930.

[45] J. Fraden, Handbook of modern sensors: physics, designs, and applications, Springer, 2004.

[46] R. Russell, Robot tactile sensing, Prentice Hall, 1990.

[47] S. Teshigawara, S. Shimizu, K. Tadakuma, M. Aiguo, M. Shimojo, M. Ishikawa, High sensitivity slip sensor using pressure conductive rubber, in: Sensors, 2009 IEEE, 2009, pp. 988-991. doi:10.1109/ICSENS.2009.5398213.

[48] J. A. Rogers, T. Someya, Y. Huang, Materials and mechanics for stretchable electronics, Science 327 (5973) (2010) 16031607. doi:10.1126/science. 1182383.

[49] A. Drimus, G. Kootstra, A. Bilberg, D. Kragic, Design of a flexible tactile sensor for classification of rigid and deformable objects, Robotics and Autonomous Systems 62 (1) (2014) 3 15, new Boundaries of Robotics. doi:http://dx.doi.org/10. $1016 / j$.robot . 2012.07.021.

[50] G. Büscher, R. Koiva, C. Schürmann, R. Haschke, H. J. Ritter, Flexible and stretchable fabric-based tactile sensor, in: IEEE/RSJ International Conference on Intelligent Robots and Systems (IROS 2012), Workshop on Advances in Tactile Sensing and Touch based Human-Robot Interaction, 2012.

[51] S. Stassi, V. Cauda, G. Canavese, C. F. Pirri, Flexible tactile sensing based on piezoresistive composites: A review, Sensors 14 (3) (2014) 5296-5332. doi:10.3390/s140305296.

[52] R. S. Dahiya, M. Valle, Tactile sensing for robotic applications, Sensors, Focus on Tactile, Force and Stress Sensors (2008) 298304.

[53] K. Weiss, H. Worn, The working principle of resistive tactile sensor cells, in: Mechatronics and Automation, 2005 IEEE International Conference, Vol. 1, 2005, pp. 471-476 Vol. 1. doi:10.1109/ICMA. 2005.1626593.

[54] G. H. Büscher, R. Kõiva, C. Schürmann, R. Haschke, H. J. Ritter, Flexible and stretchable fabric-based tactile sensor, Robotics and Autonomous Systems 63 (2015) 244-252. doi: $10.1016 / j$.robot .2014 .09 .007 .

[55] M. Shimojo, A. Namiki, M. Ishikawa, R. Makino, K. Mabuchi, A tactile sensor sheet using pressure conductive rubber with electrical-wires stitched method, Sensors Journal, IEEE 4 (5) (2004) 589-596. doi:10.1109/JSEN. 2004.833152.

[56] I. Electronics, Fsr, http://www.interlinkelectronics.com/ fsrtech.php, accessed April 29, 2014.

[57] Tekscan, Flexiforce, http://www.tekscan.com/flexiforce. html, accessed April 29, 2014.

[58] W. Robotics, Tactile sensors, http://weiss-robotics.de/en/ tactile-sensors.html, accessed April 4, 2014.

[59] L. Inaba Rubber Company, Conductive rubber, http://www. 
inaba-rubber.co.jp/en/b_products/inastomer/index.html, accessed April 29, 2014.

[60] Eeonyx, Piezoresistive fabric sensors, http://www.eeonyx . com/eeontex.php, accessed April 29, 2014.

[61] ATi, F/t sensor: Nano17, http://www.ati-ia.com/products/ ft/ft_models.aspx?id=Nano17, accessed May 9, 2014.

[62] T. Someya, T. Sekitani, Bionic skins using flexible organic devices, in: Micro Electro Mechanical Systems (MEMS), 2014 IEEE 27th International Conference on, 2014, pp. 68-71. doi : 10.1109/MEMSYS . 2014.6765575

[63] H. kew Lee, J. Chung, S.-I. Chang, E. Yoon, Normal and shear force measurement using a flexible polymer tactile sensor with embedded multiple capacitors, Microelectromechanical Systems, Journal of 17 (4) (2008) 934-942. doi:10.1109/ JMEMS . 2008.921727

[64] C. A. Jara, J. Pomares, F. A. Candelas, F. Torres, Control framework for dexterous manipulation using dynamic visual servoing and tactile sensors' feedback, Sensors 14 (1) (2014) 1787-1804. doi:10.3390/s140101787.

[65] B. Heyneman, M. Cutkosky, Biologically inspired tactile classification of object-hand and object-world interactions, in: Robotics and Biomimetics (ROBIO), 2012 IEEE International Conference on, 2012, pp. 167-173. doi:10.1109/ROBIO.2012. 6490961.

[66] PPS, Tactile sensors, http://www.pressureprofile.com/ products.php, accessed May 9, 2014.

[67] AD, Ad7147 technical datasheet, http://www.analog.com/ static/imported-files/Data_Sheets/AD7147.pdf, accessed May 9, 2014

[68] P. Maiolino, M. Maggiali, G. Cannata, G. Metta, L. Natale, A flexible and robust large scale capacitive tactile system for robots, Sensors Journal, IEEE 13 (10) (2013) 3910-3917. doi : 10.1109/JSEN. 2013.2258149.

[69] L. Seminara, M. Capurro, P. Cirillo, G. Cannata, M. Valle, Electromechanical characterization of piezoelectric $\{\mathrm{PVDF}\}$ polymer films for tactile sensors in robotics applications, Sensors and Actuators A: Physical 169 (1) (2011) 49 - 58. doi: http://dx.doi.org/10.1016/j.sna.2011.05.004

[70] S. Schulz, C. Pylatiuk, A. Kargov, R. Oberle, G. Bretthauer, Progress in the development of anthropomorphic fluidic hands for a humanoid robot, in: Humanoid Robots, 2004 4th IEEE/RAS International Conference on, Vol. 2, 2004, pp. 566-575. doi : 10.1109/ICHR . 2004.1442671.

[71] C.-H. Chuang, M.-S. Wang, Y.-C. Yu, C.-L. Mu, K.-F. Lu, C.-T. Lin, Flexible tactile sensor for the grasping control of robot fingers, in: Advanced Robotics and Intelligent Systems (ARIS), 2013 International Conference on, 2013, pp. 141-146. doi : 10.1109/ARIS. 2013.6573549.

[72] T. Zhang, H. Liu, L. Jiang, S. Fan, J. Yang, Development of a flexible 3-d tactile sensor system for anthropomorphic artificial hand, Sensors Journal, IEEE 13 (2) (2013) 510-518. doi: 10.1109/JSEN. 2012.2220345.

[73] Peratech, Quantum tunneling composite, http://www . peratech.com, accessed May 9, 2014.

[74] S. R. Company, Developments in dextrous hands for advanced robotic applications, in: Automation Congress, 2004. Proceedings. World, Vol. 15, 2004, pp. 123-128.

[75] T. B. Martin, R. Ambrose, M. Diftler, J. Platt, R., M. J. Butzer, Tactile gloves for autonomous grasping with the nasa/darpa robonaut, in: Robotics and Automation, 2004. Proceedings. ICRA '04. 2004 IEEE International Conference on, Vol. 2, 2004, pp. 1713-1718 Vol.2. doi:10.1109/ROBOT. 2004.1308071.

[76] P. Kampmann, F. Kirchner, Integration of fiber-optic sensor arrays into a multi-modal tactile sensor processing system for robotic end-effectors, Sensors 14 (4) (2014) 6854-6876. doi: $10.3390 / \mathrm{s} 140406854$.

[77] H. Xie, A. Jiang, H. Wurdemann, H. Liu, L. Seneviratne, K. Althoefer, Magnetic resonance-compatible tactile force sensor using fiber optics and vision sensor, Sensors Journal, IEEE 14 (3) (2014) 829-838. doi:10.1109/JSEN.2013.2281591.
[78] M. K. Johnson, F. Cole, A. Raj, E. H. Adelson, Microgeometry capture using an elastomeric sensor, ACM Trans. Graph. 30 (4) (2011) 46:1-46:8. doi:10.1145/2010324.1964941.

[79] R. Li, E. Adelson, Sensing and recognizing surface textures using a gelsight sensor, in: Computer Vision and Pattern Recognition (CVPR), 2013 IEEE Conference on, 2013, pp. 12411247. doi:10.1109/CVPR.2013.164.

[80] M. Koike, S. Saga, T. Okatani, K. Deguchi, Sensing method of total-internal-reflection-based tactile sensor, in: World Haptics Conference (WHC), 2011 IEEE, 2011, pp. 615-619. doi:10. 1109/WHC. 2011.5945556

[81] O. LTD., Opto-force sensor, http://www.optoforce.com/ 3dsensor/, accessed June 10, 2015.

[82] Honeywell, Bridge pressure sensor, http://sccatalog. honeywell.com/pdbdownload/images/26pc.smt. series. chart.1.pdf, accessed May 12, 2014.

[83] J. Fishel, V. Santos, G. Loeb, A robust micro-vibration sensor for biomimetic fingertips, in: Biomedical Robotics and Biomechatronics, 2008. BioRob 2008. 2nd IEEE RAS EMBS International Conference on, 2008, pp. 659-663. doi:10.1109/ BIOROB. 2008.4762917.

[84] SynTouch, The biotac, http://www.syntouchllc.com/ Products/BioTac/, accessed May 12, 2014.

[85] Y. Tenzer, L. P. Jentoft, R. D. Howe, Inexpensive and easily customized tactile array sensors using mems barometers chips, IEEE Robotics and Automation Magazine.

[86] N. Wettels, A. Parnandi, J.-H. Moon, G. Loeb, G. Sukhatme, Grip control using biomimetic tactile sensing systems, Mechatronics, IEEE/ASME Transactions on 14 (6) (2009) 718-723. doi : 10.1109/TMECH. 2009.2032686.

[87] TakkTile, Takktile kit, http://www.takktile.com, accessed Feb. 10, 2014.

[88] B. Choi, S. Lee, H. R. Choi, S. Kang, Development of anthropomorphic robot hand with tactile sensor : Skku hand ii, in: Intelligent Robots and Systems, 2006 IEEE/RSJ International Conference on, 2006, pp. 3779-3784. doi:10.1109/IROS. 2006. 281763.

[89] T. Kawamura, N. Inaguma, K. Nejigane, K. Tani, H. Yamada, Measurement of slip, force and deformation using hybrid tactile sensor system for robot hand gripping an object., International Journal of Advanced Robotic Systems 10.

[90] H. Hasegawa, Y. Mizoguchi, K. Tadakuma, A. Ming, M. Ishikawa, M. Shimojo, Development of intelligent robot hand using proximity, contact and slip sensing, in: Robotics and Automation (ICRA), 2010 IEEE International Conference on, 2010, pp. 777-784. doi:10.1109/ROBOT.2010.5509243.

[91] K. Hosoda, Y. Tada, M. Asada, Anthropomorphic robotic soft fingertip with randomly distributed receptors 54 (2) 104-109. doi:10.1016/j.robot.2005.09.019.

[92] M. Möser, Structure-borne sound, in: Engineering Acoustics, Springer Berlin Heidelberg, 2009, pp. 117-142. doi:10.1007/ 978-3-540-92723-5_4.

[93] L.-T. Jiang, J. R. Smith, Seashell effect pretouch sensing for robotic grasping, in: Robotics and Automation (ICRA), 2012 IEEE International Conference on, IEEE, pp. 2851-2858.

[94] H. Liu, J. Greco, X. Song, J. Bimbo, L. Seneviratne, K. Althoefer, Tactile image based contact shape recognition using neural network, in: Multisensor Fusion and Integration for Intelligent Systems (MFI), 2012 IEEE Conference on, 2012, pp. 138-143. doi:10.1109/MFI.2012.6343036.

[95] Y. Bekiroglu, J. Laaksonen, J. A. Jorgensen, V. Kyrki, D. Kragic, Assessing grasp stability based on learning and haptic data, Robotics, IEEE Transactions on 27 (3) (2011) 616-629. doi:10.1109/TRO.2011.2132870.

[96] Freescale, Miniature i2c digital barometer, http: //cache.freescale.com/files/sensors/doc/data_sheet/ MPL115A2.pdf, accessed May 12, 2014.

[97] M. Quigley, K. Conley, B. Gerkey, J. Faust, T. Foote, J. Leibs, R. Wheeler, A. Y. Ng, ROS: an open-source robot operating system, in: ICRA workshop on open source software, Vol. 3, 2009, p. 5 . 
[98] Shadowrobot, Shadow dexterous hand, http://www. shadowrobot.com/products/dexterous-hand/, accessed May 12, 2014.

[99] G. Metta, P. Fitzpatrick, L. Natale, YARP: yet another robot platform, International Journal on Advanced Robotics Systems 3 (1) (2006) 43-48.

[100] G. Metta, G. Sandini, D. Vernon, L. Natale, F. Nori, The icub humanoid robot: An open platform for research in embodied cognition, in: Proceedings of the 8th Workshop on Performance Metrics for Intelligent Systems, PerMIS '08, ACM, New York, NY, USA, 2008, pp. 50-56. doi:10.1145/1774674. 1774683.

[101] A. Namiki, Y. Imai, M. Ishikawa, M. Kaneko, Development of a high-speed multifingered hand system and its application to catching, in: Intelligent Robots and Systems, 2003. (IROS 2003). Proceedings. 2003 IEEE/RSJ International Conference on, Vol. 3, 2003, pp. 2666-2671 vol.3. doi:10.1109/IROS. 2003. 1249273

[102] H. Bruyninckx, P. Soetens, B. Koninckx, The real-time motion control core of the Orocos project, in: IEEE International Conference on Robotics and Automation, 2003, pp. 2766-2771.

[103] A. Schmitz, M. Maggiali, L. Natale, B. Bonino, G. Metta, A tactile sensor for the fingertips of the humanoid robot icub, in: Intelligent Robots and Systems (IROS), 2010 IEEE/RSJ International Conference on, 2010, pp. 2212-2217. doi:10. 1109/IROS. 2010.5648838.

[104] H. Liu, K. Nguyen, V. Perdereau, J. Bimbo, J. Back, M. Godden, L. Seneviratne, K. Althoefer, Finger contact sensing and the application in dexterous hand manipulation 1-17doi: 10.1007/s10514-015-9425-4.

[105] Z. Kappassov, Y. Khassanov, A. Saudabayev, A. Shintemirov, H. Varol, Semi-anthropomorphic 3d printed multigrasp hand for industrial and service robots, in: Mechatronics and Automation (ICMA), 2013 IEEE International Conference on, 2013, pp. 1697-1702. doi:10.1109/ICMA.2013.6618171.

[106] M. Diftler, C. Culbert, R. Ambrose, J. Platt, R., W. Bluethmann, Evolution of the nasa/darpa robonaut control system, in: Robotics and Automation, 2003. Proceedings. ICRA '03. IEEE International Conference on, Vol. 2, 2003, pp. 2543-2548 vol.2. doi:10.1109/ROBOT. 2003.1241975.

[107] CyberGlove, Cyberglove, http://www.cyberglovesystems . $\mathrm{com} /$, accessed May 12, 2014.

[108] Schunk, 2-finger-parallel gripper, http://www.schunk.com/ schunk_files/attachments/OM_AU_PG__EN.pdf, accessed April 29, 2014

[109] Schunk, 3-finger gripping hand sdh, http://www.schunk.com/ schunk_files/attachments/SDH_DE_EN.pdf, accessed April $29,2014$.

[110] T. Mouri, H. Kawasaki, K. Yoshikawa, J. Takai, S. Ito, Anthropomorphic robot hand: Gifu hand iii, in: Proc. Int. Conf. ICCAS, 2002, pp. 1288-1293.

[111] I. Gaiser, S. Schulz, A. Kargov, H. Klosek, A. Bierbaum, C. Pylatiuk, R. Oberle, T. Werner, T. Asfour, G. Bretthauer, R. Dillmann, A new anthropomorphic robotic hand, in: $\mathrm{Hu}-$ manoid Robots, 2008. Humanoids 2008. 8th IEEE-RAS International Conference on, 2008, pp. 418-422. doi:10.1109/ ICHR. 2008.4755987.

[112] Robotiq, 3-finger adaptive robot gripper, http://robotiq. com/en/products/industrial-robot-hand, accessed May 12, 2014.

[113] P. A. Schmidt, E. Maël, R. P. Würtz, A sensor for dynamic tactile information with applications in human-robot interaction and object exploration, Robotics and Autonomous Systems 54 (12) (2006) 1005 - 1014. doi:http://dx.doi.org/10. 1016/j.robot.2006.05.013.

[114] Barret, Barret hand, http://www.barrett.com/robot/ products-hand.htm, accessed May 12, 2014.

[115] M. Strohmayr, D. Schneider, The dlr artificial skin step ii: Scalability as a prerequisite for whole-body covers, in: Intelligent Robots and Systems (IROS), 2013 IEEE/RSJ International Conference on, 2013, pp. 4721-4728. doi:10.1109/IROS.
2013.6697036

[116] M. Grebenstein, M. Chalon, W. Friedl, S. Haddadin, T. Wimböck, G. Hirzinger, R. Siegwart, The hand of the dlr hand arm system: Designed for interaction, The International Journal of Robotics Research 31 (13) (2012) 1531-1555. doi: 10.1177/0278364912459209.

[117] K. Koyama, H. Hasegawa, Y. Suzuki, A. Ming, M. Shimojo, Pre-shaping for various objects by the robot hand equipped with resistor network structure proximity sensors, in: Intelligent Robots and Systems (IROS), 2013 IEEE/RSJ International Conference on, 2013, pp. 4027-4033. doi:10.1109/IROS . 2013.6696932

[118] Twendy-one, Twendy-one robot hand, http://twendyone.com, accessed August 4, 2014.

[119] Simlab, Allegro-hand, http://www.simlab.co.kr/ Allegro-Hand.htm, accessed August 4, 2014.

[120] K. Dautenhahn, C. L. Nehaniv, M. L. Walters, B. Robins, H. Kose-Bagci, N. A. Mirza, M. Blow, Kaspar-a minimally expressive humanoid robot for human-robot interaction research, Applied Bionics and Biomechanics 6 (3-4) (2009) 369-397.

[121] P. Mittendorfer, G. Cheng, Humanoid multimodal tactilesensing modules, Robotics, IEEE Transactions on 27 (3) (2011) 401-410. doi:10.1109/TRO.2011.2106330.

[122] Bosch, Apas assistant, http://www.bosch-apas.com/media/ en/apas/microsite_apas/2014_apasassistant.pdf, accessed May 26, 2014.

[123] S. Nicosia, RAMSETE: Articulated and Mobile Robotics for Services and Technology, Vol. 270, Springer, 2001.

[124] R. Howe, Tactile sensing and control of robotic manipualtion, Journal of Advanced Robotic 8 (3) (1994) 245-261.

[125] D. Prattichizzo, J. C. Trinkle, Grasping, in: B. Siciliano, O. Khatib (Eds.), Springer Handbook of Robotics, Springer Berlin Heidelberg, 2008, p. 671-700.

[126] F. Cordella, L. Zollo, A. Salerno, D. Accoto, E. Guglielmelli, B. Siciliano, Human hand motion analysis and synthesis of optimal power grasps for a robotic hand., International Journal of Advanced Robotic Systems 11.

[127] C. Goldfeder, P. Allen, Data-driven grasping, Autonomous Robots 31 (1) (2011) 1-20. doi:10.1007/s10514-011-9228-1.

[128] S. Ye, K. Suzuki, Y. Suzuki, M. Ishikawa, M. Shimojo, Robust robotic grasping using ir net-structure proximity sensor to handle objects with unknown position and attitude, in: Robotics and Automation (ICRA), 2013 IEEE International Conference on, 2013, pp. 3271-3278. doi:10.1109/ICRA.2013.6631033.

[129] D. Accoto, R. Sahai, F. Damiani, D. Campolo, E. Guglielmelli, P. Dario, A slip sensor for biorobotic applications using a hot wire anemometry approach, Sensors and Actuators A: Physical 187 (0) (2012) 201 - 208. doi:http://dx.doi.org/10.1016/ j.sna.2008.07.030.

[130] R. Howe, M. Cutkosky, Sensing skin acceleration for slip and texture perception, in: Robotics and Automation, 1989. Proceedings., 1989 IEEE International Conference on, 1989, pp. 145-150 vol.1. doi:10.1109/ROBOT.1989.99981.

[131] A. Cranny, D. Cotton, P. Chappell, S. Beeby, N. White, Thickfilm force and slip sensors for a prosthetic hand, Sensors and Actuators A: Physical 123-124 (0) (2005) 162 - 171, eurosensors $\{$ XVIII 2004 The 18th European conference on SolidState Transducers.

[132] J. T. Belter, J. L. Segil, A. M. Dollar, R. F. Weir, Mechanical design and performance specifications of anthropomorphic prosthetic hands: A review., Journal of Rehabilitation Research \& Development 50 (5).

[133] V. A. Ho, T. Nagatani, A. Noda, S. Hirai, What can be inferred from a tactile arrayed sensor in autonomous in-hand manipulation?, in: Automation Science and Engineering (CASE), 2012 IEEE International Conference on, 2012, pp. 461-468. doi:10.1109/CoASE. 2012.6386384.

[134] J. J. Benedetto, Wavelets: mathematics and applications, Vol. 13, CRC press, 1993

[135] A. Vezhnevets, Gml adaboost matlab toolbox, http://graphics.cs.msu.ru/en/science/research/ 
machinelearning/adaboosttoolbox, accessed May 26, 2014.

[136] C.-C. Chang, C.-J. L. (2001), Libsvm: A library for support vector machines, http://www.csie.ntu.edu.tw/ cjlin/ libsvm/, accessed May 26, 2014

[137] K. Murphy, Hidden markov model toolbox for matlab, http:// www.cs.ubc.ca/ murphyk/Software/HMM/hmm.html, accessed May 26, 2014.

[138] X. Wu, V. Kumar, J. R. Quinlan, J. Ghosh, Q. Yang, H. Motoda, G. J. McLachlan, A. Ng, B. Liu, S. Y. Philip, et al., Top 10 algorithms in data mining, Knowledge and Information Systems 14 (1) (2008) 1-37.

[139] T. Araki, T. Nakamura, T. Nagai, K. Funakoshi, M. Nakano, N. Iwahashi, Online object categorization using multimodal information autonomously acquired by a mobile robot, Advanced Robotics 26 (17) (2012) 1995-2020. doi:10.1080/01691864. 2012.728693.

[140] H. Liu, X. Song, J. Bimbo, L. Seneviratne, K. Althoefer, Surface material recognition through haptic exploration using an intelligent contact sensing finger, in: Intelligent Robots and Systems (IROS), 2012 IEEE/RSJ International Conference on, 2012, pp. 52-57. doi:10.1109/IROS.2012.6385815.

[141] J. Sinapov, V. Sukhoy, R. Sahai, A. Stoytchev, Vibrotactile recognition and categorization of surfaces by a humanoid robot, Robotics, IEEE Transactions on 27 (3) (2011) 488-497. doi:10.1109/TRO.2011.2127130.

[142] N. Jamali, C. Sammut, Majority voting: Material classification by tactile sensing using surface texture, Robotics, IEEE Transactions on 27 (3) (2011) 508-521. doi:10.1109/TRO. 2011. 2127110.

[143] P. Dallaire, P. Giguère, D. Émond, B. Chaib-draa, Autonomous tactile perception: A combined improved sensing and bayesian nonparametric approach, Robotics and Autonomous Systems 62 (4) (2014) 422-435. doi:dx.doi.org/ $10.1016 / j$. robot .2013 .11 .011 .

[144] R. Ibrayev, Y.-B. Jia, Recognition of curved surfaces from onedimensional tactile data, Automation Science and Engineering, IEEE Transactions on 9 (3) (2012) 613-621. doi:10.1109/ TASE. 2012.2194143.

[145] A. Schneider, J. Sturm, C. Stachniss, M. Reisert, H. Burkhardt, W. Burgard, Object identification with tactile sensors using bag-of-features, in: Intelligent Robots and Systems, 2009. IROS 2009. IEEE/RSJ International Conference on, 2009, pp. 243-248. doi:10.1109/IROS.2009.5354648.

[146] S. Navarro, N. Gorges, H. Worn, J. Schill, T. Asfour, R. Dillmann, Haptic object recognition for multi-fingered robot hands, in: Haptics Symposium (HAPTICS), 2012 IEEE, 2012, pp. 497-502. doi : 10.1109/HAPTIC. 2012.6183837.

[147] S. Luo, W. Mou, M. Li, K. Althoefer, H. Liu, Rotation and translation invariant object recognition with a tactile sensor, in: SENSORS, 2014 IEEE, 2014, pp. 1030-1033. doi :10.1109/ ICSENS. 2014.6985179.

[148] H. Liu, X. Song, T. Nanayakkara, L. D. Seneviratne, K. Althoefer, A computationally fast algorithm for local contact shape and pose classification using a tactile array sensor, in: Robotics and Automation (ICRA), 2012 IEEE International Conference on, 2012, pp. 1410-1415. doi:10.1109/ICRA.2012. 6224872 .

[149] R. Li, R. Platt, W. Yuan, A. ten Pas, N. Roscup, M. Srinivasan, E. Adelson, Localization and manipulation of small parts using gelsight tactile sensing, in: Intelligent Robots and Systems (IROS 2014), 2014 IEEE/RSJ International Conference on, 2014, pp. 3988-3993. doi:10.1109/IROS.2014. 6943123.

[150] H. Yan, M. H. Ang, A. N. Poo, A survey on perception methods for human-robot interaction in social robots, International Journal of Social Robotics 6 (1) (2014) 85-119. doi : $10.1007 /$ s12369-013-0199-6.

[151] N. Wettels, G. Loeb, Haptic feature extraction from a biomimetic tactile sensor: Force, contact location and curvature, in: Robotics and Biomimetics (ROBIO), 2011 IEEE
International Conference on, 2011, pp. 2471-2478. doi:10. 1109/ROBIO.2011.6181676

[152] J. Matas, C. Galambos, J. Kittler, Robust detection of lines using the progressive probabilistic hough transform, Comput. Vis. Image Underst. 78 (1) (2000) 119-137. doi:10.1006/ cviu.1999.0831.

[153] S. Hutchinson, G. Hager, P. Corke, A tutorial on visual servo control, Robotics and Automation, IEEE Transactions on 12 (5) (1996) 651-670. doi:10.1109/70.538972.

[154] S. H. François Chaumette, Visual servoing and visual tracking, in: B. Siciliano, O. Khatib (Eds.), Springer Handbook of Robotics, Springer, 2008.

[155] A. D. Berger, P. K. Khosla, Using tactile data for realtime feedback, The International Journal of Robotics Research 10 (2) (1991) 88-102. doi:10.1177/027836499101000202.

[156] P. Sikka, H. Zhang, S. Sutphen, Tactile servo: Control of touch-driven robot motion, in: Experimental Robotics III, Springer, 1994, pp. 219-233.

[157] A. Okamura, N. Smaby, M. Cutkosky, An overview of dexterous manipulation, in: Robotics and Automation, 2000. Proceedings. ICRA '00. IEEE International Conference on, Vol. 1, 2000, pp. 255-262 vol.1. doi:10.1109/ROBOT.2000.844067.

[158] H. Zhang, N. Chen, Control of contact via tactile sensing, Robotics and Automation, IEEE Transactions on 16 (5) (2000) 482-495. doi:10.1109/70.880799.

[159] N. Chen, H. Zhang, R. Rink, Edge tracking using tactile servo, in: Intelligent Robots and Systems 95.'Human Robot Interaction and Cooperative Robots', Proceedings. 1995 IEEE/RSJ International Conference on, Vol. 2, IEEE, 1995, pp. 84-89.

[160] J. Back, J. Bimbo, Y. Noh, L. Seneviratne, K. Althoefer, H. Liu, Control a contact sensing finger for surface haptic exploration, in: Robotics and Automation (ICRA), 2014 IEEE International Conference on, 2014, pp. 2736-2741. doi : 10.1109/ICRA. 2014.6907251

[161] J. Bimbo, L. Seneviratne, K. Althoefer, H. Liu, Combining touch and vision for the estimation of an object's pose during manipulation, in: Intelligent Robots and Systems (IROS), 2013 IEEE/RSJ International Conference on, 2013, pp. 40214026. doi:10.1109/IROS. 2013.6696931.

[162] M. H. Raibert, J. J. Craig, Hybrid position/force control of manipulators, Journal of Dynamic Systems, Measurement, and Control 103 (2) (1981) 126-133. 